Working Paper 14-02

Statistics and Econometrics Series 02

February 2014
Departamento de Estadística

Universidad Carlos III de Madrid

Calle Madrid, 126

28903 Getafe (Spain)

Fax (34) 91 624-98-49

\title{
THE UNCERTAINTY OF CONDITIONAL RETURNS, VOLATILITIES AND CORRELATIONS IN DCC MODELS
}

\author{
Diego E Fresoli* and Esther Ruiz ${ }^{\dagger *}$
}

\begin{abstract}
When forecasting conditional correlations that evolve according to a Dynamic Conditional Correlation (DCC) model, only point forecasts can be obtained at each moment of time. In this paper, we analyze the finite sample properties of a bootstrap procedure to approximate the density of these forecasts that also allows obtaining conditional densities for future returns and volatilities. The procedure is illustrated by obtaining conditional forecast intervals and regions of returns, volatilities and correlations in the context of a system of daily exchange rates returns of the Euro, Japanese Yen and Australian Dollar against the US Dollar.
\end{abstract}

Keywords: Bootstrap forecast intervals, Dynamic Conditional Correlation, Exchange rates, Forecast regions, Realized correlation, Resampling methods.

* Dpt. de Estadística, Universidad Carlos III de Madrid, C/ Madrid 126, 28903 Getafe (Madrid), Spain, e-mail: dfresoli@est-econ.uc3m.es. ${ }^{\dagger}$ Dpto. Estadística and Instituto Flores de Lemus, Universidad Carlos III de Madrid. ${ }^{\star}$ Acknowledgements: Both authors acknowledge financial support from the Spanish Government project ECO2012-32401. Also, Gian Piero Aielli helped us with the estimation of cDCC models. 


\title{
The uncertainty of conditional returns, volatilities and correlations in DCC models
}

\author{
Diego Fresoli*and Esther Ruiz ${ }^{\dagger \ddagger}$
}

February 2014

\begin{abstract}
When forecasting conditional correlations that evolve according to a Dynamic Conditional Correlation (DCC) model, only point forecasts can be obtained at each moment of time. In this paper, we analyze the finite sample properties of a bootstrap procedure to approximate the density of these forecasts that also allows obtaining conditional densities for future returns and volatilities. The procedure is illustrated by obtaining conditional forecast intervals and regions of returns, volatilities and correlations in the context of a system of daily exchange rates returns of the Euro, Japanese Yen and Australian Dollar against the US Dollar.

KEY WORDS: Bootstrap forecast intervals, Dynamic Conditional Correlation, Exchange rates, Forecast regions, Realized correlation, Resampling methods.
\end{abstract}

\section{Introduction}

Forecasting conditional correlations is a key issue for financial market participants when dealing, for example, with risk management, derivative pricing models, hedging strategies or portfolio allocation models; see, for example, details in Engle (2009). Multivariate GARCH (MGARCH) models are also widely implemented to macroeconomic time series. Modeling the time variation in macroeconomic volatility is important to the accuracy of inference and to explain, for example, the sources of the Great Moderation; see, for example, Clark and Ravazzolo (in press) for a very recent reference. Moreover, MGARCH are becoming popular when modeling non-financial time series as, for example, wind speed; see Jeon and Taylor (2012) among others. As a result, modeling the second order moments of multivariate time series has become a prevailing field of research and many MGARCH models have been developed with this purpose; see Bauwens et al. (2006) and Silvennoinen and Teräsvirta (2009) for comprehensive surveys. Among the many MGARCH models available in the literature, the Dynamic Conditional Correlation (DCC) model of Engle (2002) has become one of the most popular models for the estimation of conditional

\footnotetext{
*Corresponding author: Dpt. de Estadística, Universidad Carlos III de Madrid, C/ Madrid 126, 28903 Getafe (Madrid), Spain, e-mail: dfresoli@est-econ.uc3m.es.

${ }^{\dagger}$ Dpto. Estadística and Instituto Flores de Lemus, Universidad Carlos III de Madrid.

\#cknowledgements. Both authors acknowledge financial support from the Spanish Government project ECO2012-32401. Also, Gian Piero Aielli helped us with the estimation of cDCC models.
} 
correlations; see Audrino (in press) and Laurent et al. (2012) for the forecasting usefulness of DCC models. The DCC model assumes that the dynamic evolution of the pairwise correlations is similar to that of univariate GARCH conditional variances. Recently, Aielli (2013) proposes a reformulation of the original specification of the correlation driving process so that it is possible to obtain a consistent Quasi-Maximum Likelihood (QML) estimator of the parameters, giving rise to the corrected Dynamic Conditional Correlation ( $\mathrm{cDCC}$ ) model. Note that the consistency of the sample estimator of the unconditional correlations is crucial for the adequate performance of the bootstrap procedure considered in this paper. The cDCC model is now the bechmark for empirical applications in the context of multivariate conditional heterocedastic times series of financial returns and it will be the focus of this paper; see, for example, Engle and Kelly (2012), Hafner and Reznikova (2012), Bauwens et al. (2013), Aielli and Caporin (in press) and Audrino (in press) for applications of the cDCC model.

It is important to note that the conditional correlations in CDCC models are observable onestep-ahead; see Caporin and McAleer (2013) who suggest that, in spite of its limitations, the DCC model can be considered as a filter for estimating and forecasting conditional correlations. Consequently, the only uncertainty associated with one-step-ahead correlations is that attributable to the parameter estimation. However, when the correlations are forecast more than one-step-ahead, they also have uncertainty associated with the future forecast errors. As far as we know, there have been no attempts in the literature to measure the uncertainty associated with conditional correlations forecast by DCC models; see, for example, Laurent et al. (2012) and Caporin and McAleer (2010) who compare point forecasts from various MGARCH models without even mentioning the associated uncertainty. The only attempts of measuring the uncertainty of conditional correlations appear in the context of high frequency realized correlations, where Barndorff-Nielsen and Shephard (2004) provide asymptotic intervals and Dovonon et al. (2013) propose bootstrap intervals.

In the context of univariate GARCH models, Pascual et al. (2006) propose using bootstrap procedures to obtain forecast densities of returns and conditional volatilities; see Hartz et al. (2006), Gaglianone et al. (2011), Grigoletto and Lisi (2011), Huang and Wang (2012) and Wang et al. (2012) for empirical implementations. In a multivariate setting, Fresoli et al. (2014) illustrate how to adapt this bootstrap procedure to obtain forecast distributions of the correlations in the context of a VAR-DCC model. The proposed bootstrap procedure is appealing because it allows the construction of multivariate forecast densities for returns, volatilities and correlations that incorporate the parameter uncertainty without relying on any particular assumption about the 
distribution of standardized returns. This paper analyzes the finite sample performance of the bootstrap procedure described by Fresoli et al. (2014) when implemented to obtain conditional forecast regions for future returns, volatilities and correlations in the context of the cDCC model. It is important to remark that this bootstrap procedure can be easily adapted to deal with other MGARCH models. We illustrate the procedure by implementing it to construct time-varying forecast Bonferroni regions for returns and forecast intervals for volatilities and correlations in a three-dimensional system containing daily exchange rate returns of the Euro, Japanese Yen and Australian Dollar against the US Dollar.

The rest of this paper is structured as follows. Section 2 describes the cDCC model and the bootstrap algorithm proposed to approximate the conditional forecast densities of returns, volatilities and correlations. In Section 3, we carry out Monte Carlo experiments to analyze the finite sample properties of the bootstrap procedure. In Section 4, the bootstrap algorithm is implemented to forecast returns, volatilities and correlations of a three-dimensional system of daily exchange rates returns. Finally, Section 5 concludes the paper.

\section{Bootstrap densities for conditional correlations}

In this section, we establish briefly notation by describing the scalar cDCC model. The bootstrap procedure proposed by Fresoli et al. (2014) to obtain forecast densities of returns, volatilities and correlations in the context of the cDCC model is also described.

\subsection{The DCC model}

The scalar cDCC model as originally proposed by Engle (2002) and modified by Aielli (2013) is given by

$$
\begin{gathered}
y_{t}=H_{t}^{1 / 2} a_{t} \\
H_{t}=D_{t} R_{t} D_{t}
\end{gathered}
$$

where $y_{t}$ is a $K \times 1$ vector of returns observed at time $t, a_{t}$ is a $K \times 1$ serially independent vector with zero mean and identity covariance matrix, $H_{t}$ is a $K \times K$ positive definite conditional covariance matrix, $D_{t}$ is a $K \times K$ diagonal matrix containing the univariate GARCH-type conditional standard deviations of each of the variables in $y_{t}$ and $R_{t}$ is the $K \times K$ matrix of conditional correlations. The most popular model for univariate conditional variances is the basic $\operatorname{GARCH}(1,1)$ 
of Engle (1982) and Bollerslev (1986) given by

$$
\sigma_{i, t}^{2}=\omega_{i}+\alpha_{i} y_{i, t-1}^{2}+\beta_{i} \sigma_{i, t-1}^{2}, \quad i=1, \ldots, K
$$

where $\sigma_{i, t}$ is the $i$ th element in the main diagonal of $D_{t}$. The parameters satisfy the conditions for positivity and weak stationarity of $y_{i, t}$; see Teräsvirta (2009) for a recent survey on univariate GARCH models. ${ }^{1}$

The correlation matrix $R_{t}$ is defined as follows

$$
R_{t}=d g\left(Q_{t}\right)^{-\frac{1}{2}} Q_{t} d g\left(Q_{t}\right)^{-\frac{1}{2}}
$$

where $d g\left(Q_{t}\right)$ is a diagonal matrix with the same diagonal elements as $Q_{t}$ which determines the dynamics of the conditional correlations as follows

$$
Q_{t}=(1-\alpha-\beta) S+\alpha v_{t-1} v_{t-1}^{\prime}+\beta Q_{t-1}
$$

where $v_{t}=d g\left(Q_{t}\right)^{\frac{1}{2}} \varepsilon_{t}$ with $\varepsilon_{t}=D_{t}^{-1} y_{t}$ being the vector of standardized errors, $S$ is the unconditional correlation matrix of $v_{t}$ and $\alpha$ and $\beta$ are scalars. The scalar $\mathrm{cDCC}$ model in equations (1)-(5) restricts the dynamics of all the correlations to be governed by the same parameters, namely $\alpha$ and $\beta$. Furthermore, positiveness is guaranteed if $S$ is positive definite and $\alpha, \beta>0$ and $\alpha+\beta<1$.

The cDCC model delivers point forecasts of correlations and volatilities. The $h$-steps-ahead forecast of the volatility of each return in $y_{t}$ is obtained easily by iterating forward as follows

$$
\sigma_{i, T+h \mid T}^{2}=\omega_{i} \sum_{j=1}^{h-2}\left(\alpha_{i}+\beta_{i}\right)^{j}+\left(\alpha_{i}+\beta_{i}\right)^{h-1} \sigma_{i, T+1 \mid T}^{2}
$$

where the one-step-ahead forecast of the conditional variance is determined by the observed series of returns as follows

$$
\sigma_{i, T+1 \mid T}^{2}=\frac{\omega_{i}}{1-\alpha_{i}-\beta_{i}}+\alpha_{i} \sum_{j=0}^{T-1} \beta_{i}^{j}\left(y_{i, T-j}^{2}-\frac{\omega_{i}}{1-\alpha_{i}-\beta_{i}}\right) .
$$

\footnotetext{
${ }^{1}$ For simplicity, we focus on the simplest $\mathrm{cDCC}(1,1)$ specification with $\operatorname{GARCH}(1,1)$ conditional variances.
} 
Similarly, the point forecast of the conditional correlation matrix $R_{T+h}$ is given by

$$
R_{T+h \mid T}=d g\left(Q_{T+h \mid T}\right)^{-\frac{1}{2}} Q_{T+h \mid T} d g\left(Q_{T+h \mid T}\right)^{-\frac{1}{2}} .
$$

where

$$
Q_{T+h \mid T}=(1-\alpha-\beta) S \sum_{j=0}^{h-2}(\alpha+\beta)^{j}+(\alpha+\beta)^{h-1} Q_{T+1 \mid T}
$$

and

$$
Q_{T+1 \mid T}=S+\alpha \sum_{j=0}^{T-1} \beta^{j}\left[\left(d g\left(Q_{T-j}\right)^{-\frac{1}{2}} D_{T-j}^{-1} y_{T-j} y_{T-j}^{\prime} D_{T-j}^{-1} d g\left(Q_{T-j}\right)^{-\frac{1}{2}}-S\right] .\right.
$$

Using $D_{T+h \mid T}$ and $R_{T+h \mid T}$, it is possible to construct $H_{T+h \mid T}$ using equation (2). Finally, assuming further that $\varepsilon_{t}$ is conditionally Gaussian, one can approximate the joint forecast density of $y_{T+h}$ as follows

$$
y_{T+h} \mid y_{1}, \ldots, y_{T} \sim N\left(0, H_{T+h \mid T}\right) .
$$

Note that, even if the errors were truly Gaussian, the forecast density in (11) is only valid for $h=1$ and it should be considered as an approximation for $h>1$; see Drost and Nijman (1993) in the context of univariate GARCH models. In any case, in practice, standardized financial returns often depart from Gaussianity usually due to fat-tails; see, for instance, Bauwens and Laurent (2005), Pesaran and Pesaran (2007) and Rossi and Spazzini (2010). If the errors are non-Gaussian, future densities predicted as in (11) might be a poor approximation of the conditional distribution of returns.

Furthermore, the parameters in equations (6) and (9) are unknown and must be estimated using, for example, the three-steps correlation target estimator described by Engle (2009) and Aielli (2013); see Hafner and Reznikova (2012) for a comparison of alternative estimators of DCC models. Aielli (2013) derives heuristically the asymptotic distribution of the cDCC three-steps correlation target estimator and shows that it is consistent and asymptotically Gaussian under standard assumptions. The density in (11) does not incorporate the parameter uncertainty and, consequently, it will underestimate the uncertainty associated with future returns.

Finally, note that equations (6) and (9) only yield point forecasts of future volatilities and correlations. The bootstrap procedure described next allows constructing forecast densities for future returns, volatilities and correlations that incorporate the parameter uncertainty without relying on specific assumptions on the error distribution. 


\subsection{Bootstrap forecasts in the cDCC model}

Fresoli et al. (2014) propose a bootstrap procedure to approximate the forecast density of future returns, volatilities and correlations for the DCC model. In this section, we describe how to adapt this procedure to forecast in the context of the $\mathrm{cDCC}$ model.

Denote by $\theta=\left(\Upsilon_{1}, \Upsilon_{2}, s\right)$ where $\Upsilon_{1}=\left[\left(\omega_{1}, \alpha_{1}, \beta_{1}\right), \ldots,\left(\omega_{K}, \alpha_{K}, \beta_{K}\right)\right]$ is a vector containing the parameters in the conditional variances, $\Upsilon_{2}=(\alpha, \beta)$ is a vector whose components are the parameters that govern the dynamics in the conditional correlation, and $s=v e c h(S)$, is a vector stacking the lower off-diagonal elements of $S$, with vech being the off-diagonal stacking operator. The algorithm to obtain bootstrap replicates of returns, volatilities and correlations is as follows.

Step 1. Estimate the model parameters $\theta$ by the three-steps correlation target procedure as described by Aielli (2013). Denote the estimated parameter by $\widehat{\theta}$. Obtain $\widehat{a}_{t}=\widehat{H}_{t}^{-\frac{1}{2}} y_{t}$ and the corresponding empirical distribution function denoted by $\widehat{F}_{\widehat{a}}$.

Step 2. Compute $Q_{1}^{*}=\widehat{S}$ and the corresponding correlation matrix $R_{1}^{*}=d g\left(Q_{1}^{*}\right)^{-\frac{1}{2}} Q_{1}^{*} d g\left(Q_{1}^{*}\right)^{-\frac{1}{2}}$. Obtain $\varepsilon_{1}^{*}=R_{1}^{* \frac{1}{2}} a_{1}^{*}$ and $v_{1}^{*}=d g\left(Q_{1}^{*}\right)^{\frac{1}{2}} \varepsilon_{1}^{*}$, where $a_{1}^{*}$ is a random draw with replacement from $\widehat{F}_{\widehat{a}}$. Construct recursively for $t=2, \ldots, T$, a bootstrap replicate of $\varepsilon_{t}^{*}, v_{t}^{*}$ and $R_{t}^{*}$ as follows

$$
\begin{gathered}
Q_{t}^{*}=(1-\widehat{\alpha}-\widehat{\beta}) \widehat{S}+\widehat{\alpha} v_{t-1}^{*} v_{t-1}^{* \prime}+\widehat{\beta} Q_{t-1}^{*}, \\
R_{t}^{*}=d g\left(Q_{t}^{*}\right)^{-\frac{1}{2}} Q_{t}^{*} d g\left(Q_{t}^{*}\right)^{-\frac{1}{2}}, \\
\varepsilon_{t}^{*}=R_{t}^{* \frac{1}{2}} a_{t}^{*}, \\
v_{t}^{*}=d g\left(Q_{t}^{*}\right)^{\frac{1}{2}} \varepsilon_{t}^{*},
\end{gathered}
$$

where $a_{t}^{*}$ are random draws with replacement from $\widehat{F}_{\widehat{a}}$. Consider $\sigma_{i, 1}^{* 2}=\widehat{\omega}_{i} /\left(1-\widehat{\alpha}_{i}-\widehat{\beta}_{i}\right)$ and $y_{i, 1}^{*}=\varepsilon_{i, 1}^{*} \sigma_{i, 1}^{*}$, for $i=1, \ldots, K$. Obtain recursively for $t=2, \ldots, T$, a bootstrap replicate of $y_{t}$ and their conditional variances as follows

$$
\begin{gathered}
\sigma_{i, t}^{2 *}=\widehat{\omega}_{i}+\widehat{\alpha}_{i} y_{i, t-1}^{* 2}+\widehat{\beta}_{i} \sigma_{i, t-1}^{* 2}, \\
y_{i, t}^{*}=\varepsilon_{i, t}^{*} \sigma_{i, t}^{*},
\end{gathered}
$$

where $\varepsilon_{i, t}^{*}$ is the $i$ th element of $\varepsilon_{t}^{*}$ obtained in (12).

Step 3. Obtain a bootstrap estimate of the parameters $\widehat{\theta}^{*}$ by fitting the cDCC model to 
the bootstrap replicate $\left\{y_{1}^{*}, \ldots, y_{T}^{*}\right\}$. Construct $\widehat{D}_{t}^{*}$ and $\widehat{Q}_{t}^{*}$, for $t=1, \ldots, T$, which contain the in-sample estimates of the univariate conditional standard deviations and correlations obtained by using the bootstrap estimates of the parameters and the returns series as follows

$$
\begin{gathered}
\widehat{\sigma}_{i, t}^{*}=\left[\widehat{\omega}_{i}^{*}+\widehat{\alpha}_{i}^{*} y_{i, t-1}^{2}+\widehat{\beta}_{i}^{*} \widehat{\sigma}_{i, t-1}^{* 2}\right]^{\frac{1}{2}}, \\
\widehat{Q}_{t}^{*}=\left(1-\widehat{\alpha}^{*}-\widehat{\beta}^{*}\right) \widehat{S}^{*}+\widehat{\alpha}^{*}\left[d g\left(\widehat{Q}_{t-1}^{* \frac{1}{2}}\right) \widehat{D}_{t-1}^{*-1} y_{t-1} y_{t-1}^{\prime} \widehat{D}_{t-1}^{*-1} d g\left(\widehat{Q}_{t-1}^{* \frac{1}{2}}\right)\right]+\widehat{\beta}^{*} \widehat{Q}_{t-1}^{*} .
\end{gathered}
$$

Step 4. Compute one-step-ahead bootstrap forecasts of conditional correlations, variances and returns according to

$$
\begin{gathered}
Q_{T+1 \mid T}^{*}=\widehat{S}^{*}+\widehat{\alpha}^{*} \sum_{j=0}^{T-1} \widehat{\beta}^{* j}\left[d g\left(\widehat{Q}_{T-j}^{*}\right)^{\frac{1}{2}} \widehat{D}_{T-j}^{*-1} y_{T-j} y_{T-j}^{\prime} \widehat{D}_{T-j}^{*-1} d g\left(\widehat{Q}_{T-j}^{*}\right)^{\frac{1}{2}}-\widehat{S}^{*}\right] \\
R_{T+1 \mid T}^{*}=d g\left(Q_{T+1 \mid T}^{*}\right)^{-\frac{1}{2}} Q_{T+1 \mid T}^{*} d g\left(Q_{T+1 \mid T}^{*}\right)^{-\frac{1}{2}} \\
\varepsilon_{T+1}^{*}=R_{T+1 \mid T}^{* \frac{1}{2}} a_{T+1}^{*}, \\
v_{T+1}^{*}=d g\left(Q_{T+1 \mid T}^{*}\right)^{\frac{1}{2}} \varepsilon_{T+1}^{*} \\
\widehat{\sigma}_{i, T+1 \mid T}^{2 *}=\frac{\widehat{\omega}_{i}^{*}}{1-\widehat{\alpha}_{i}^{*}-\widehat{\beta}_{i}^{*}}+\widehat{\alpha}_{i}^{*} \sum_{j=0}^{T-1} \widehat{\beta}_{i}^{* j}\left(y_{i, T-j}^{2}-\frac{\widehat{\omega}_{i}^{*}}{1-\widehat{\alpha}_{i}^{*}-\widehat{\beta}_{i}^{*}}\right) \\
y_{i, T+1}^{*}=\varepsilon_{i, T+1}^{*} \sigma_{i, T+1 \mid T}^{*} .
\end{gathered}
$$

where $a_{T+1}^{*}$ is a random draw with replacement from $\widehat{F}_{\widehat{a}}$. Likewise, obtain future values of the correlations, volatilities and returns through the following recursion for $h=2, \ldots, H$

$$
\begin{gathered}
Q_{T+h \mid T}^{*}=\left(1-\widehat{\alpha}^{*}-\widehat{\beta}^{*}\right) \widehat{S}^{*}+\widehat{\alpha}^{*} v_{T+h-1}^{*} v_{T+h-1}^{* \prime}+\widehat{\beta}^{*} Q_{T+h-1 \mid T}^{*}, \\
R_{T+h \mid T}^{*}=d g\left(Q_{T+h \mid T}^{*}\right)^{-\frac{1}{2}} Q_{T+h \mid T}^{*} d g\left(Q_{T+h \mid T}^{*}\right)^{-\frac{1}{2}} \\
\varepsilon_{T+h}^{*}=R_{T+h \mid T}^{* \frac{1}{2}} a_{T+h}^{*} \\
v_{T+h}^{*}=d g\left(Q_{T+h \mid T}^{*}\right)^{\frac{1}{2}} \varepsilon_{T+h}^{*} \\
\sigma_{i, T+h \mid T}^{2 *} \widehat{\omega}_{i}^{*}+\widehat{\alpha}_{i}^{*} y_{i, T+h-1}^{* 2}+\widehat{\beta}_{i}^{*} \sigma_{i, T+h-1 \mid T}^{* 2} \\
y_{i, T+h}^{*}=\varepsilon_{i, T+h}^{*} \sigma_{i, T+h \mid T}^{*} .
\end{gathered}
$$

where $a_{T+h}^{*}$ are random draws with replacement from $\widehat{F}_{\widehat{a}}$. 
Step 5. Repeat steps 2 to $4, B$ times, obtaining $y_{T+h \mid T}^{*(b)}, D_{T+h \mid T}^{*(b)}$ and $R_{T+h \mid T}^{*(b)}$ for $h=1, \ldots, H$ and $b=1, \ldots, B$.

It is worth mentioning that the one-step-ahead forecast of $Q_{t}$ in (18) depends on $\widehat{D}_{t}^{*}$ and $\widehat{Q}_{t}^{*}$, which are the in-sample estimates of $D_{t}$ and $Q_{t}$ evaluated at the bootstrap estimates of the parameters, $\widehat{\theta}^{*}$, and the original observations. Therefore, although the one-step-ahead bootstrap forecasts of the correlation matrix is conditional on the observed sample, their variability is affected by the parameter uncertainty. The only component which varies from one bootstrap replicate to another is the bootstrap estimates of the parameters, $\widehat{\theta}^{*}$, while $\left\{y_{1}, \ldots, y_{T}\right\}$ are kept fixed throughout all bootstrap replicates. When forecasting further into the future, the bootstrap forecasts of the correlation matrix also includes the error uncertainty by sampling with replacement from $\widehat{F}_{\widehat{a}}$ to obtain $a_{T+h}^{*(b)}$. A similar argument holds for the bootstrap forecasts of the conditional variances; see also the discussion in Pascual et al. (2006) for a similar argument in the univariate context.

Using the bootstrap replicates, one can obtain estimates of the multivariate forecast densities of returns, volatilities and correlations. Also, it is possible to obtain their forecast regions and intervals. Consider first the construction of time-varying forecast regions for returns. First, we can obtain bootstrap forecast ellipsoids with probability content $(1-\alpha) 100 \%$ which are given by

$$
B E_{y}(T+h)=\left\{y \mid y \in\left[y_{T+h}-\overline{\widehat{y}_{T+h \mid T}^{*}}\right] H_{T+h \mid T}^{*-1}\left[y_{T+h}-\overline{\widehat{y}_{T+h \mid T}^{*}}\right]<C^{*}\right\}
$$

where $\overline{\widehat{y}_{T+h}^{*}}$ is the sample mean of the $B$ bootstrap replicates of returns $\widehat{y}_{T+h}^{*(b)}, H_{T+h \mid T}^{*}$ is the corresponding sample covariance of $y_{T+h}^{*}$, and $C^{*}$ is the $(1-\alpha) 100 \%$ percentile of the bootstrap distribution of the following quadratic form

$$
\left[\widehat{y}_{T+h}^{*(b)}-\overline{\widehat{y}_{T+h}^{*(b)}}\right] H_{T+h \mid T}^{*(b)-1}\left[\widehat{y}_{T+h}^{*(b)}-\overline{\widehat{y}_{T+h}^{*(b)}}\right]
$$

It is well-known that the ellipsoids are only appropriate when the distribution is Gaussian; see Fresoli et al. (2014) and Wolf and Wunderli (2012). Furthermore, constructing ellipsoids can be computationally complicated when the dimension of the system is very large. Alternatively, one can construct Bonferroni cubes with probability content at least $(1-\alpha) 100 \%$ for future returns 
which are given by

$$
B C_{y}(T+h)=\left\{y \mid y \in \cup_{j=1}^{K}\left[q_{j, T+h}^{y *}\left(\frac{\alpha}{2 K}\right), q_{j, T+h}^{y *}\left(1-\frac{\alpha}{2 K}\right)\right]\right\}
$$

where $q_{j, T+h}^{y *}(\zeta)$ is the $\zeta$ th quantile of the empirical bootstrap distribution of the $j$ th return, $G_{y_{j, T+h}}^{*}(x)=\#\left(y_{j, T+h \mid T}^{*(b)} \leq x\right) / B$. As the Bonferroni cubes do not offer information about the association between returns, they can be corrected to be expressed in the direction of the correlations; see Fresoli et al. (2014).

As an illustration, we consider several bivariate cDCC models obtained combining three sets of correlation parameters, $\Upsilon_{2}=(0.18,0.70),(0.1,0.88)$ and $(0.03,0.95)$, two error distributions, namely Gaussian and Student-7, two values for the unconditional correlation, 0.5 and 0.9 , and univariate GARCH models with parameters $\Upsilon_{1}=(0.05,0.05,0.90,0.01,0.10,0.85)$. So we consider 12 different models. For each model we generate a time series of size $T=1000$ and estimate the cDCC model parameters by the three-steps correlation target estimator. ${ }^{2}$ Then, the bootstrap procedure is implemented with $B=1000$ bootstrap replicates to obtain one-steps-ahead forecast densities of returns. We also obtain the corresponding Gaussian forecast densities computed as in equation (11) with $H_{T+1 \mid T}$ substituted by $\widehat{H}_{T+1 \mid T}$. From each of these conditional densities, we construct the $95 \%$ one-step-ahead ellipsoids and corrected Bonferroni cubes. The results are plotted in Figure 1 for the three models with Gaussian errors and unconditional correlation equal to 0.5 for three selected within-sample periods. In each, a realization of 1000 returns, $y_{T+1}$, has been represented by dots. A quick inspection of all panels in Figure 1 reveals that, for a particular model, both the shape and the slope of the ellipsoids and Bonferroni cubes change over time. Also, it is noticeable that the bootstrap ellipsoids have larger volumes than the corresponding Gaussian ellipsoids and, in some cases, the true observation is included in the former but not in the latter. The discrepancy between bootstrap and standard regions is even more pronounced for the corrected Bonferroni cubes. Given that the models considered in Figure 1 have Gaussian errors and the forecast horizon is one, the differences between the regions based on the Gaussianity assumption and the bootstrap regions can be attributed to the parameter uncertainty.

\footnotetext{
${ }^{2}$ The computations have been carried out by using a MATLAB code developed by the first author in a workstation with processor Intel Core i5-2.50GHz and 8GB of RAM. Correlation target estimates are obtained numerically by using fminunc with the interior point algorithm. For each $\operatorname{GARCH}(1,1)$ process, the intercept, $\omega_{i}$, is restricted to be greater than $e^{-6}$ while the elements in $S$ are obtained as centered correlations. All other parameters, $\alpha_{i}, \beta_{i}, \alpha$ and $\beta$, are restricted to lie in the interval $\left(e^{-6}, 1-e^{-6}\right)$ with $\alpha_{i}+\beta_{i}<1$ and $\alpha+\beta<1$ being less than $1-e^{-6}$.
} 


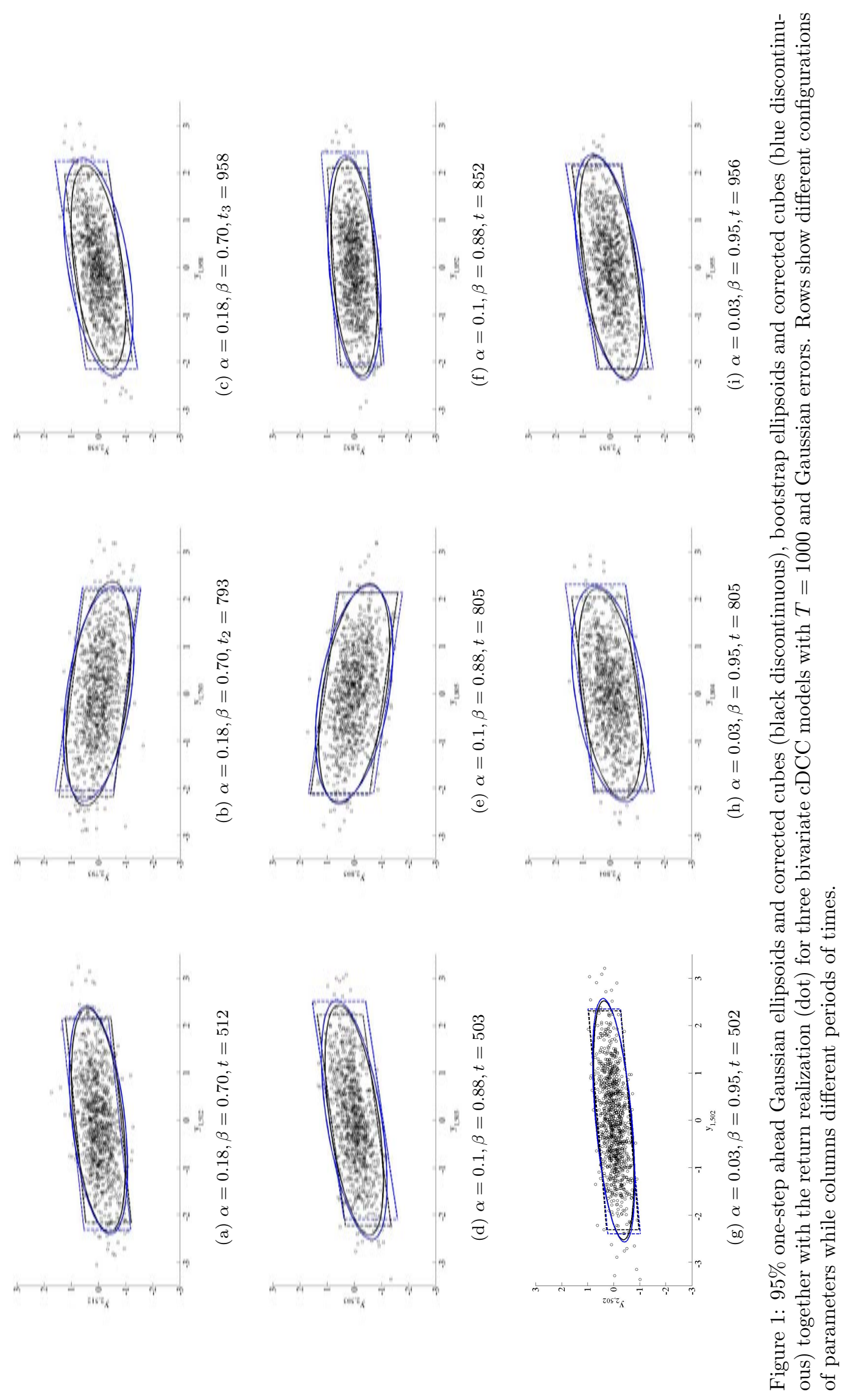


Following the same ideas as in Pascual et al. (2006) for univariate conditional variances, we can use the bootstrap replicates of the $j$ th univariate conditional variance to construct its empirical bootstrap distribution, $G_{\sigma_{j, T+h}^{2}}^{*}(x)=\#\left(\sigma_{j, T+h \mid T}^{2 *(b)} \leq x\right) / B$. For completeness we also illustrate the construction of forecast densities for conditional variances. Given that, in the cDCC model, the conditional variances are assumed to be contemporaneously independent, here we only consider the construction of the (1- $\alpha) 100 \%$ marginal forecast intervals which are given by

$$
B I_{\sigma^{2}}(T+h)=\left\{\sigma^{2} \mid \sigma^{2} \in\left[q_{j, T+h}^{\sigma^{2} *}\left(\frac{\alpha}{2}\right), q_{j, T+h}^{\sigma^{2} *}\left(1-\frac{\alpha}{2}\right)\right]\right\}
$$

where $q_{j, T+h}^{\sigma^{2} *}(\zeta)=G_{\sigma_{j, T+h}^{2}}^{*-1}$ is the $\zeta$ th percentile of the empirical bootstrap distribution of the $j$ th conditional variance. To illustrate the construction of bootstrap forecast densities and their corresponding forecast intervals for conditional variances, we consider a bivariate time series generated by the $\mathrm{cDCC}$ with correlation parameters $\Upsilon_{2}=(0.03,0.95)$, the unconditional correlation being 0.9 and the error distribution being Student-7. For the sake of presentation, we just focus on the conditional variance for the second variable in the system. Figure 2 displays kernel estimates of the $h$-steps-ahead bootstrap conditional densities together with the empirical conditional densities of $\sigma_{2, T+h}^{2}$, for $h=1,2$ and 20. The empirical densities have been obtained using a kernel estimate implemented to 2000 simulated future values of the series, conditional on $\left\{y_{1}, \ldots, y_{T}\right\}$. First, observe that the one-step-ahead forecast of $\sigma_{2, T+1}^{2}$ plotted in panel (a) of Figure 2 is concentrated on a fixed value. As we stated before, no uncertainty is associated with forecasting one-step-ahead conditional variances. Yet the conditional bootstrap distribution of $\sigma_{2, T+1}^{2}$ has some degree of variability reflecting the parameter uncertainty. Second, note that the conditional 2-steps-ahead forecast of $\sigma_{2, T+2}^{2}$ is bounded by the value of the intercept, $\omega_{2}$ and, consequently, the empirical density is asymmetric to the right. However, the bootstrap replicates, $\sigma_{2, T+2}^{2 *}$, might be smaller than this value depending on the bootstrap estimates of the parameters, $\left(\widehat{\omega}_{2}^{*}, \widehat{\alpha}_{2}^{*}, \widehat{\beta}_{2}^{*}\right)$. Third, the uncertainty associated with $\sigma_{2, T+h}^{2}$ clearly increases as we forecast further into the future due to the addition of error uncertainty, a fact that is reflected by both, the empirical and the bootstrap conditional distributions of $\sigma_{2, T+h}^{2}$. After all, the first three panels of Figure 2 display bootstrap densities that approximate quite well the true empirical ones with the approximation being better for longer forecast horizon than short ones. This is due to the fact that the model implies known one-step-ahead forecast.

Panel (d) of Figure 2 plots one realization of $\sigma_{2, T+h}^{2}$, for $h=1, \ldots, 20$, together with the corresponding point forecasts and the $95 \%$ bootstrap intervals. It can be observed that the true 


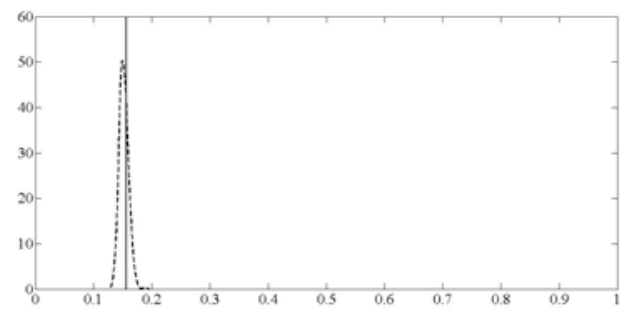

(a) $\sigma_{2, T+1}^{2}$

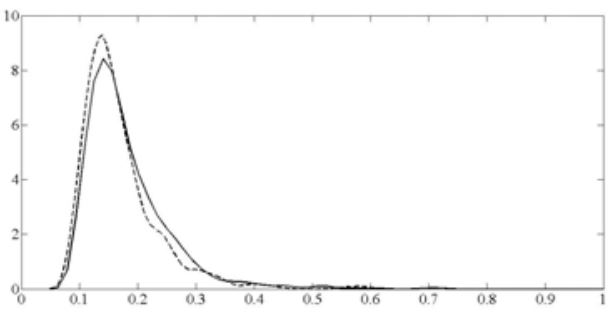

(c) $\sigma_{2, T+20}^{2}$

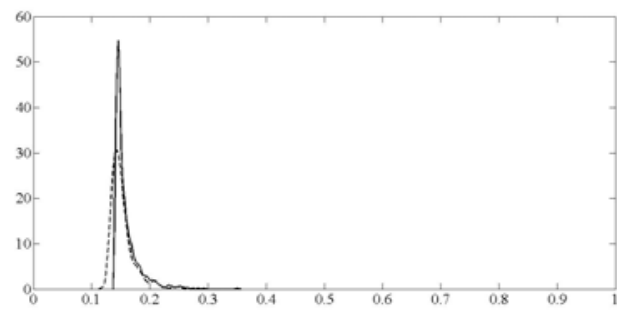

(b) $\sigma_{2, T+2}^{2}$

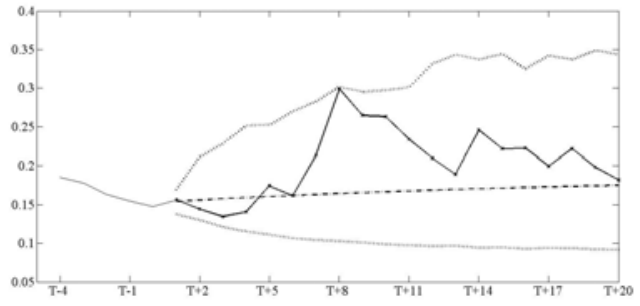

(d) $\sigma_{2, T+h}^{2}$

Figure 2: Kernel estimates of empirical (continuous line) and bootstrap densities (discontinuous line) of $\sigma_{2, T+h}^{2}$ within the bivariate cDCC model with sample size $T=1000$ and Student-7 errors for (a) $h=1$, (b) $h=2$ (c) $h=20$ (d) $95 \% h$-steps-ahead forecast interval, for $h=$ $1, \ldots, 20$, (discontinous lines) together with point forecasts (dash-dot line) and out-of-sample true realization (continuous line).

out-of-sample realizations of the volatilities are included within the bootstrap forecast intervals.

Finally, we implement the bootstrap procedure to construct $h$-steps-ahead bootstrap forecast densities for conditional correlations. Panels (a), (b) and (c) of Figure 3 plot kernel estimates of the conditional empirical $h$-steps-ahead forecast densities of $\rho_{12, T+h}$, for $h=1,2$ and 20 together with kernel estimates of the corresponding bootstrap densities for the bivariate cDCC model, with correlation parameters $\Upsilon_{2}=(0.10,0.88)$, unconditional correlation equal to 0.9 and Student-7 errors. The conclusions are similar to those obtained when forecasting volatilities. However, because, in this particular illustration, the marginal correlation is close to one, the densities are asymmetric to the left. After all, it is clear from the first three Panels of Figure 3 that the bootstrap procedure it approximates adequately the shape of the conditional empirical densities of $\rho_{12, T+h}$.

Once more, the $h$-steps-ahead bootstrap densities can be used to construct the corresponding intervals for the forecasts of the correlations. Panel (d) of Figure 3 plots an out-of-sample realization of the correlations, $\rho_{12, T+h}$, for $h=1,2 \ldots, 20$, together with the point forecasts obtained after estimating the model and the $95 \%$ bootstrap forecast intervals. We observe that the true correlations fall within the bounds of the forecast intervals. Further, we observe that the point 
forecasts underestimate the corresponding the out-of-sample correlations; see Engle and Sheppard (2001).

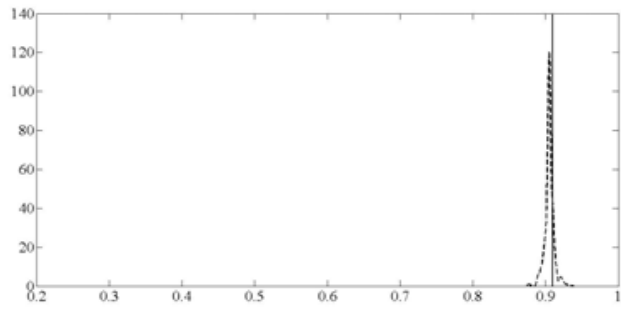

(a) $\rho_{12, T+1}$

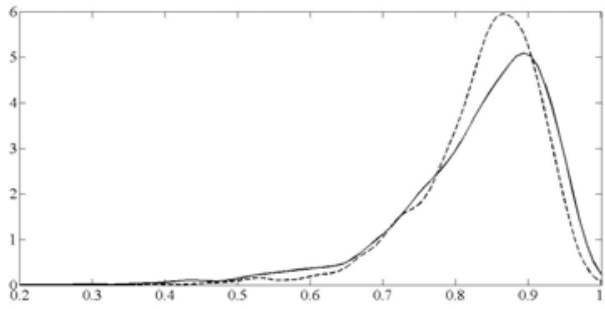

(c) $\rho_{12, T+20}$

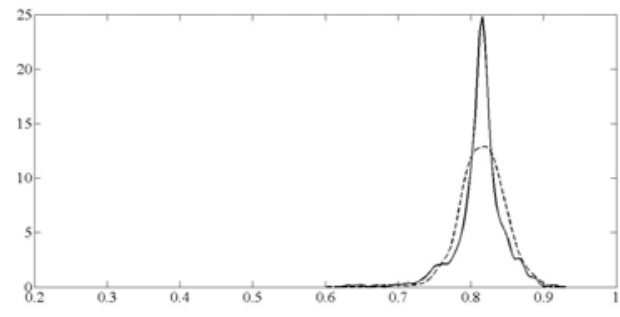

(b) $\rho_{12, T+2}$

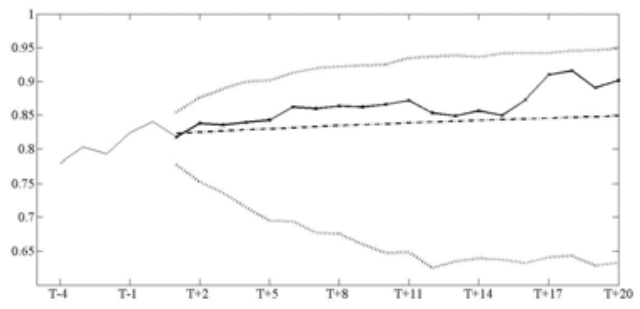

(d) $\rho_{12, T+h}$

Figure 3: Kernel estimates of empirical densities (continuous line) and bootstrap densities (discontinuous line) of $\rho_{12, T+h}$ within of a bivariate cDCC with $T=1000$ and Student-7 error for (a) $h=1$, (b) $h=2$ (c) $h=20$ (d) $95 \% h$-steps-ahead forecast interval, for $h=1, \ldots, 20$, (discontinuous lines) together with point forecasts (dash dot line) and out-of-sample true realization (continuous line).

\section{Monte Carlo evidence}

In this section, we carry out Monte Carlo experiments to analyse the finite sample properties of the bootstrap procedure described in the previous section. We focus on two of the bivariate $\mathrm{cDCC}$ models, used for illustration in the previous section. The first model (model 1) has $\Upsilon_{2}=(0.10,0.88)$ and an unconditional correlation of 0.5 while the second model (model 2) has $\Upsilon_{2}=(0.03,0.95)$ with an unconditional correlation of 0.9 . Note that these two models bring up two different situations. In both models the persistence is 0.98 . However, the first model is characterized by a large response-to-shock parameter, $\alpha$ while the second model resembles closely the parameters found when fitting cDCC models to financial series, with $\alpha$ being relatively small; see, for example, the empirical application in next section. Three alternative distributions for $a_{t}$ are assumed, namely, Gaussian, Student- 7 and $\chi_{5}^{2}$. The second distribution is chosen to reproduce the heavy tails often observed in the distribution of standardized financial returns while the third 
is chosen to represent potential asymmetries. The sample sizes considered are $T=500,1000$ and 2000. The number of Monte Carlo replicates is 500; see Hafner and Franses (2009) and Hafner and Reznikova (2012) for similar number of Monte Carlo replications. For each replicate, we generate 2000 conditional future values of $y_{T+h}, \sigma_{T+h}^{2}$ and $\rho_{12, T+h}$, for $h=1, \ldots, 20$, to approximate the empirical distribution of returns, conditional variances and correlations, respectively. The parameters are estimated by the three-steps correlation target estimator of Aielli (2013). The number of bootstrap replicates is $B=1000$, and these are used to construct $95 \% h$-steps-ahead bootstrap forecast intervals for each return, conditional variance and correlation in the system. In order to assess the small sample properties of the bootstrap procedure, the empirical coverage is computed by counting the number of future values inside the corresponding intervals. In addition, to measure the adequacy of the bootstrap distributions to approximate the out-of-sample distributions of returns, we compute the Earth Mover's Distance (EMD) between the $h$-step-ahead bootstrap distribution of the $i$ th return and the corresponding empirical distribution. Also, we compute the EMD when the $h$-steps-ahead distribution of the $i$ th return is approximated by assuming Normality; see Rubner et al. (1998) for the definition of the EMD and Arroyo and Maté (2009) for an application of the EDM to forecast histograms. ${ }^{3}$

Figure 4 displays the empirical coverages of the $95 \%$ bootstrap forecast intervals for $y_{2, T+h}$, $\sigma_{2, T+h}^{2}$ and $\rho_{12, T+h}$ when considering model 1.

First, we consider the empirical coverages of $y_{2, T+h}$ which are plotted in the first row of Figure 4. Although the empirical coverages of the bootstrap forecast intervals are below the nominal of $95 \%$, they are always above $94.5 \%$, and this happen irrespective of the forecast horizon. We also observe that, as the sample size increases, the coverage rates of the bootstrap intervals are closer to the nominal, a feature that is more evident when the error distribution departs from Gaussianity. For the purpose of comparing alternative methods, the first row of figure Figure 4 also includes the coverage rates of the Gaussian forecast intervals. As expected, the difference between the Gaussian and bootstrap intervals are less pronounced when the error distribution is Gaussian. In contrast, in the case of the Student-7 error distribution, the coverage rates of the Gaussian intervals are well below the nominal as well as below the bootstrap coverages for all sample sizes. Surprisingly, when the error distribution is $\chi_{5}^{2}$, the Gaussian intervals provide empirical coverages above the nominal, though this distortion seems to dampen with the forecast

\footnotetext{
${ }^{3}$ The EMD can be approximated as follows. Let $x_{(1)} \leq \ldots \leq x_{(B)}$ and $y_{(1)} \leq \ldots \leq y_{(B)}$ be ordered realizations of $X$ and $Y$. Then the EMD is given by $E M D_{x y}=\left[\frac{1}{N} \sum_{i=1}^{R}\left|x_{(i)}-y_{(i)}\right|^{2}\right]^{\frac{1}{2}}$, which is just the $L^{2}$ distance between the ordered vectors. We are thankful to Ruben Zamar for suggesting using this measure.
} 
horizon.

The coverages plotted in the first row of Figure 4 are not informative about whether the shape of the density of returns is well approximated by the bootstrap density. This is why, we also compute the EMD. Figure 5 plots the average EMD between the Gaussian and the bootstrap conditional densities of $y_{2, t}$ with respect to the corresponding empirical density, when the sample size is $T=500$. Consider first the results when the errors are Gaussian. In this case, we can observe that the performance of the Gaussian and the bootstrap densities is similar for $h=1$. Note that, in this case, the true forecast density is Normal. However, if $h>1$, the forecast densities are not Normal and the EMDs of the Gaussian densities increase with $h$ and are clearly larger than the EMDs of the bootstrap densities. Therefore, even if the errors are truly Gaussian, it is worth to implement the bootstrap procedure to forecast more than one-step-ahead. On the other hand, when the errors are either Student- 7 or $\chi_{5}^{2}$, the bootstrap EMDs are clearly smaller than the EMDs of the Gaussian densities.

The small sample properties of bootstrap forecast intervals for univariate $\operatorname{GARCH}(1,1)$ processes have studied in detail by Pascual et al. (2006). In this paper, however, we also display some results regarding average coverages of the conditional variance of $y_{2, t}$. The second row of Figure 4 plots the empirical coverages of the bootstrap forecast intervals of $\sigma_{2, T+h}^{2}$ for Gaussian, Student-7 and $\chi_{5}^{2}$ errors in model 1 . We can observe that the coverages of the bootstrap forecast intervals depend on the forecast horizon. Regardless of the error distribution, the coverages are below the nominal of $95 \%$ when $h=1$. This undercoverage is more pronounced when the errors are non-Gaussian and the sample size is small. In any case, it is important to note that the coverages are always over 0.9. When $h>2$, the coverages tend to decrease as the forecast horizon increases. We can observe that, as expected, the coverages are closer to the nominal as $T$ increases suggesting that the procedure is consistent. For instance, when $T=2000$, the coverages are below the nominal by less than $2.5 \%$, and this happen for all the forecast horizons and error distributions; these results are qualitatively similar to those reported by Pascual et al. (2006), though there is a slight difference in the decline of the coverage curve as the forecast horizon increases. 

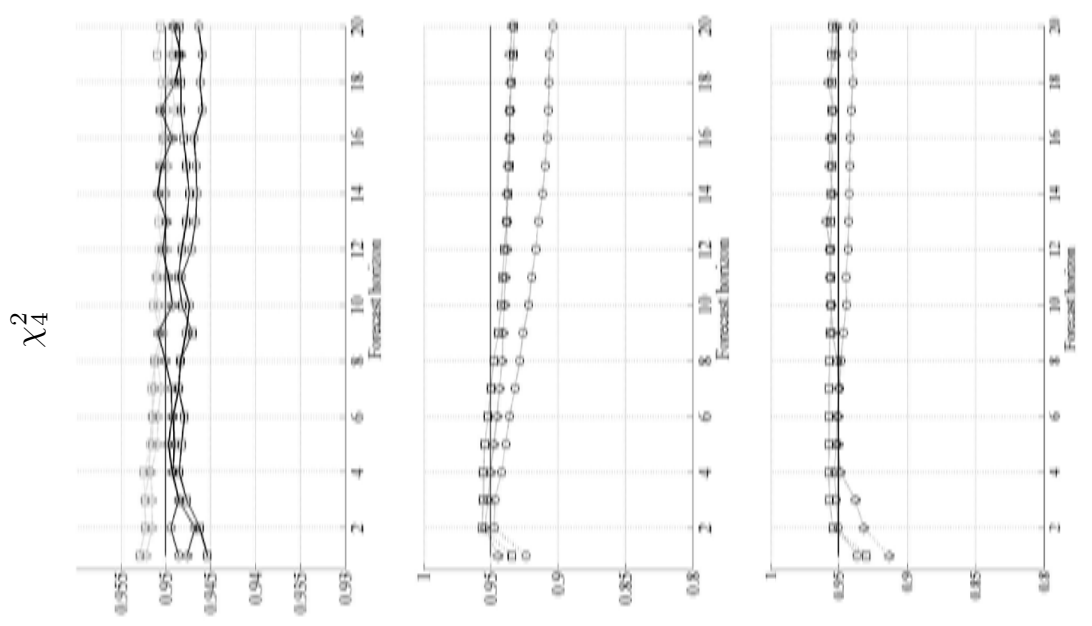

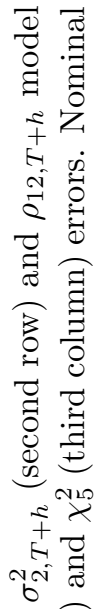

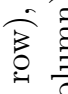

记
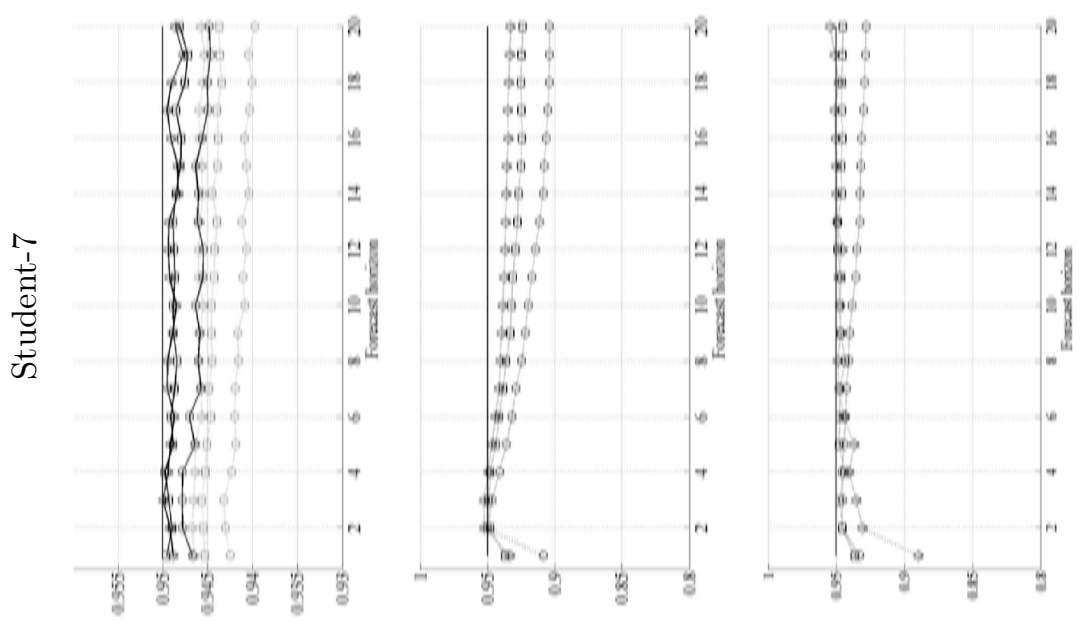

+

กิ่

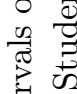

.્さ

范

包

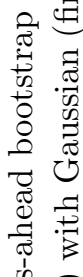

这全

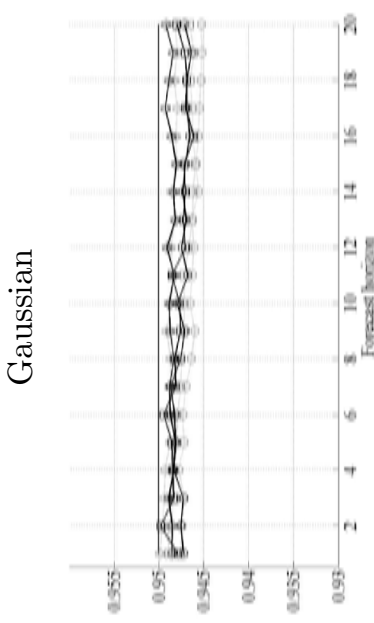

$y+L^{\prime} z h$
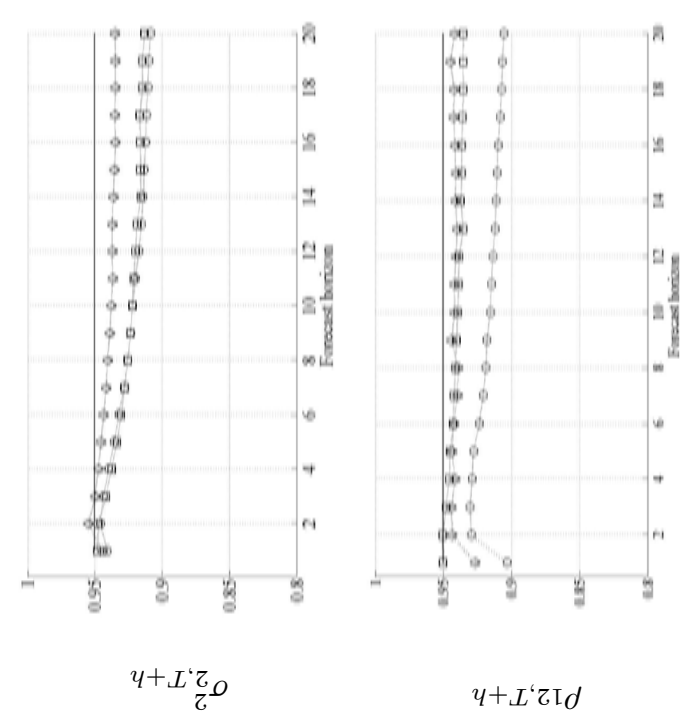

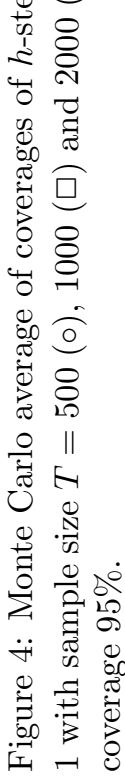




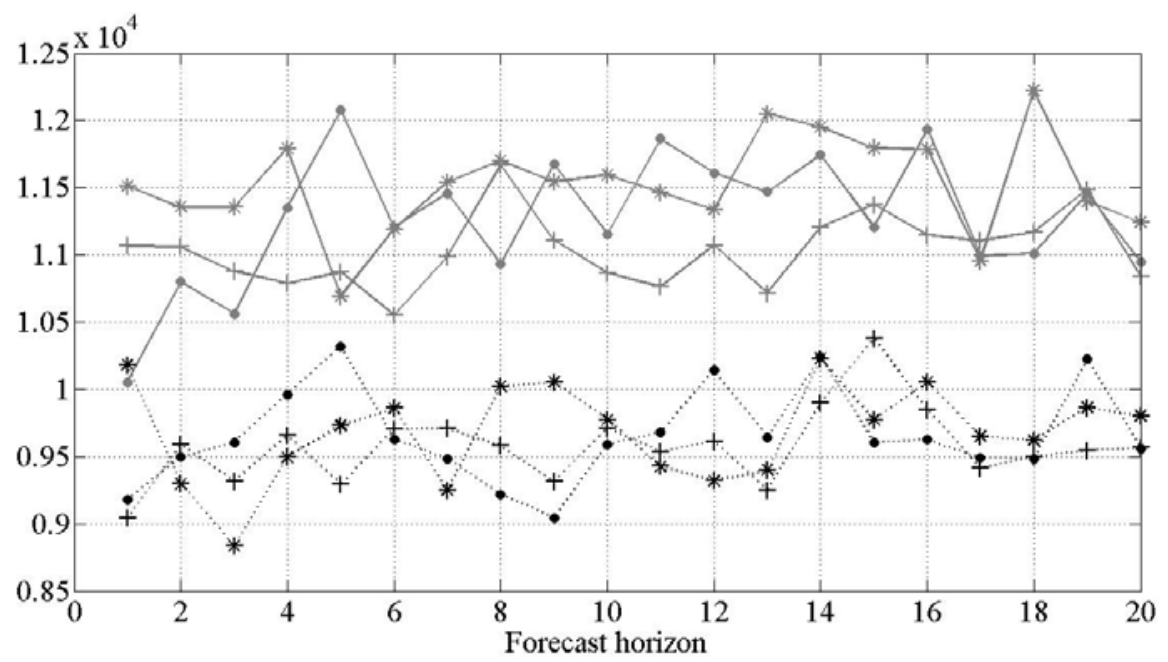

Figure 5: Monte Carlo averages of EMD distances between the $h$-steps-ahead Gaussian forecast densities (grey lines) and bootstrap densities (black lines) of $y_{2, T+h}$ and the corresponding empirical conditional density for $y_{2, t}$ in model 1 with $T=500$ and Gaussian $(\bullet)$, Student-7 (+) and $\chi_{5}^{2}(*)$ errors.

The third row of Figure 4 plots the Monte Carlo coverages of the bootstrap forecast intervals for the conditional correlation, $\rho_{12, t}$ in model 1 when the nominal coverage is $95 \%$. The patterns observed are roughly similar to those observed for the conditional variance of $y_{2, t}$. Once more, the coverages of the one-step-ahead bootstrap forecast are under the nominal for all error distribution and sample sizes. This undercoverage is slightly larger than that observed for conditional variances. When $h \geq 2$, we also observe that the coverages decrease with the forecast horizon. However, this undercoverage is only observed for the smallest sample size when $T=500$. When $T=1000$ and 2000 , the coverages of the bootstrap forecast intervals of $\rho_{12, t}$ are approximately equal to the nominal.

Finally, Figure 6 plots the empirical coverages of the bootstrap forecast intervals for $y_{1, T+h}$, $\sigma_{1, T+h}, \rho_{12, T+h}$ and average EMD between the Gaussian forecast densities and bootstrap densities of $y_{1, T+h}$ and the corresponding empirical density, when $T=500$ and the error distribution is Student-7, in model 2. Much of what we commented about Figures 4 and 5 is still valid for Figure 6. In panel (a) of Figure 6, we observe that the empirical coverages of the bootstrap forecast intervals of $y_{1, T+h}$ are below the nominal. Also, bootstrap intervals of $y_{1, T+h}$ improve their coverage with the sample size and they provide better accuracy than the corresponding Gaussian intervals for moderate and large sample sizes. Moreover, panel (d) shows that the bootstrap densities are clearly closer to the corresponding true density than the Gaussian densities when the error distributions depart from Gaussianity. Still, when the error distribution is Gaussian, the 


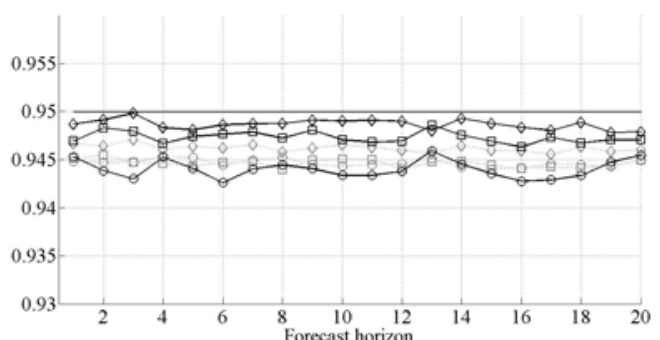

(a) $\rho_{12, T+1}$

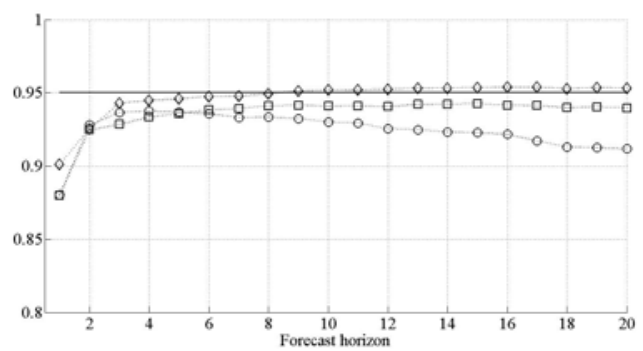

(c) $\rho_{12, T+h}$

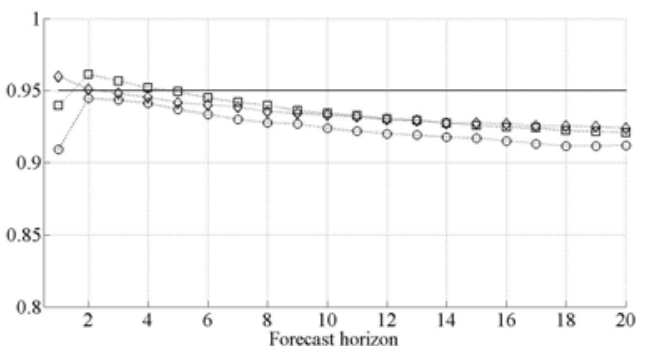

(b) $\rho_{12, T+20}$

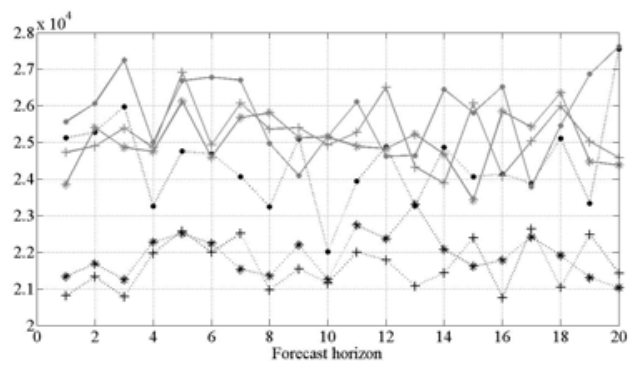

(d) $\rho_{12, T+2}$

Figure 6: Monte Carlo average of coverages of $h$-steps-ahead bootstrap forecast intervals of (a) $y_{2, T+h}$, (b) $\sigma_{2, T+h}^{2}$ and (c) $\rho_{12, T+h}$ model 2 with sample size $T=500(\circ), 1000(\square)$ and $2000(\diamond)$ with Student-7 errors and nominal coverage 95\%, and (d) averages of EMD distances between the $h$-steps-ahead Gaussian forecast densities (grey lines) and bootstrap densities (black lines)of $y_{1, T+h}$ and the corresponding empirical conditional density for $y_{1, t}$ in model 1 with $T=500$ and Gaussian $(\bullet)$, Student-7 $(+)$ and $\chi_{5}^{2}(*)$ errors. 
bootstrap seems to be as good as the Gaussian density when $h=1$. Panel (b) and (c) that plot the empirical coverages $\sigma_{1, T+h}$ and $\rho 12, T+h$, respectively, look qualitatively similar to those plotted in the second and third rows of Figures 4. Quantitatively, comparing the empirical coverages of Figures 4 and Figure 6 for $\rho 12, T+h$ we observe that are slightly closer to the nominal in model 1 than in model 2. This seems to be caused by the greater uncertainty encountered when $\alpha$ is larger relative to the $\beta$. In such a case, the the bootstrap forecast intervals become considerably wide and, thus, they are more likely to capture future realization of the conditional correlation.

After all, the Monte Carlo simulation results show that the bootstrap algorithm can reasonably deal with forecasting returns, volatilities and correlations cDCC models.

\section{Empirical application}

In this section, the bootstrap algorithm is implemented to forecast future returns, volatilities and correlations of a system of three currencies, Euro (EUR), Japanese Yen (JPY) and Australian Dollar (AUD) against the US Dollar (USD). Note that this section is purely illustrative of the application of the bootstrap procedure and limited attention is given to the economic interpretation of the results. Daily exchange rates of the three currencies, $P_{i, t} i=1,2$ and 3 , have been observed from 3/1/2003 to 13/9/2013 with 2693 observations. The full sample period have been split in a estimation period spanning from $3 / 1 / 2003$ to $1 / 8 / 2013$ with $T=2663$ and a forecast out-of-sample period from $2 / 8 / 2013$ to $13 / 9 / 2013$ with $H=30$. The currencies are transformed to log-returns as usual by $y_{i, t}=100 \times \triangle \log \left(P_{i, t}\right)$. Several descriptive statistics are reported in Table 1 for the overall period as well as for the estimation and out-of-sample periods. Note that, for all three currencies, the univariate and multivariate skewness and excess kurtosis are significantly different from zero. Therefore, each of them has a distribution that departs from Normality, a fact which is in line with the results reported by Normality test suggested by Fiorentini et al. (2004) which rejects the null hypothesis of Normality for each of the series. Table 1 also reports the Ljung-Box statistics adjusted to account for conditional heteroscedasticity as proposed by Diebold (1988) and denoted by $Q(l)$, for $l=10$. It may be observed that the returns show no linear dependence. However, squared returns are characterized by significant correlations according to the Ljung-Box statistics of order 10 for the squared returns, $Q_{2}(10)$. Finally, the last column of Table 1 reports the Ljung-Box statistics for the first ten cross-correlations between returns and future squared returns, $Q_{12}(10)$, suggesting the presence of leverage effect only in the case of AUD. 
From a multivariate point of view, the sample pairwise unconditional correlations for the EUR-AUD and EUR-JPY are 0.63 and 0.25 , respectively. However, the unconditional correlation between AUD and JPY is not different from zero. To test for constant conditional correlations, we implement the test suggested by Engle and Sheppard (2001) which considers the null hypothesis $H_{0}: R_{t}=R$ against the alternative $H_{a}: \operatorname{vech}\left(R_{t}\right)=\phi_{0}+\phi_{1} \operatorname{vech}\left(R_{t-1}\right)+\cdots+\phi_{q} \operatorname{vech}\left(R_{t-l}\right)$, where $\phi_{j}$ are scalar. Note that the alternative states the current correlation matrix depends on its own lags up an order $l$ but does no specify a cDCC process for the correlation matrix. The test statistic based on $l=10$ is 271.32 with p-value equal to 0.00 . Therefore, the constant correlation hypothesis is rejected. Next we test for asymmetries in conditional correlations, implementing the exceedance test of Hong et al. (2007) which is based on the exceedance correlations above or below a threshold. For instance, when the threshold is the median of each exchange rate return, the exceedance test statistics are 0.30, 0.53 and 0.17 for EUR-JPY, EUR-AUD and JPY-AUD, respectively, with the corresponding p-values being all larger than 0.40. Considering different thresholds does not change this result. Therefore, the presence of asymmetries in correlations is rejected.

The results above suggest fitting the symmetric cDCC model. ${ }^{4}$ Table 2 reports the threesteps target correlation estimates of the parameters. Observe that all parameters are significant and close to the values usually estimated when the $\mathrm{cDCC}$ model is fitted to systems of financial returns. Also, observe that the constant of the equation of the correlation between the Japanese Yen and the Australian Dollar exchange rate returns is not significantly different from zero when $\alpha=1 \%$. Table 2 also reports the Ljung-Box statistics for the univariate squared standardized residuals and the multivariate Ljung-Box statistic, $Q_{M}(m)$ computed for $\widehat{a}_{t}=\widehat{R}_{t}^{-\frac{1}{2}} \widehat{\varepsilon}_{t}$, which is conjectured to be distributed asymptotically as $\chi^{2}$ with $m N^{2}$ degrees of freedom; see Hafner and Franses (2009) who consider it as a measure of residual autocorrelation. The null hypothesis of no further dynamics in squared returns is not rejected. Therefore, the cDCC model seems to be adequate to represent the dynamic dependence in the system of exchange rate returns.

Using the bootstrap replicates of the parameters and the original observations, we obtain onestep-ahead densities of volatilities and correlations using equations (16) and (17). We also obtain one-step-ahead bootstrap densities of returns. We also obtain one-step-ahead bootstrap densities of $y_{t}$. Figure 7 plots the one-step-ahead forecasts and the $95 \%$ intervals for the period between $2 / 7 / 2006$ and $1 / 8 / 2013$ together with the sample estimate of the unconditional correlation. We

\footnotetext{
${ }^{4}$ Further analysis show that the presence of leverage effect in AUD is rather weak.
} 
can observe that the intervals are rather tiny so that the forecasts of correlations are very reliable. The second column of Figure 7 highlights this conclusion by plotting the one-step-ahead forecasts of the correlations for the last nineteen days, covering the period from $8 / 7 / 2013$ to $1 / 8 / 2013$. We can observe large and frequent changes in the EUR-JPY and JPY-AUD conditional correlations while the correlations between EUR-AUD are more stable. Judging from what is observed in Figure 7, the movements in EUR-AUD unconditional correlation happen closer to its unconditional correlation than those of the others correlations. As a result, one may conclude that EUR-AUD is more likely to fall within the one-step-ahead bootstrap interval than EUR-JPY and JPY-AUD correlations. To illustrate this feature, we compute the coverage rates of the one-steps-ahead bootstrap interval with respect to the unconditional correlation. The results show that, with a coverage rate of $3.56 \%$, the EUR-AUD unconditional correlation is more likely to fall within the bootstrap interval than the EUR-JPY and JPY-AUD, with coverages nearly $1.30 \%$. Similarly, although the unconditional correlation between AUD and JPY is not significantly different from zero, only $1.04 \%$ of the bootstrap intervals contains the zero within its bounds. However, the conditional correlations fluctuate around zero and this is why one can wrongly conclude that the unconditional correlation between Japanese Yen and the Australian Dollar exchange rates is zero.

The proposed bootstrap procedure is also implemented to forecast returns, volatilities and correlations out-of-sample. Figure 8 plots $h$-steps-ahead Gaussian and bootstrap forecast ellipsoids and cubes for the exchange rates returns considered two-by-two and $h=1,20$ and 30 . In each of the figures, the true observed return is represented by a dot. First, we observe that the Gaussian and bootstrap ellipsoids are very similar. This result is not surprising as the means and conditional covariances used to construct them do not differ significantly. Consider, for example, the EUR and AUD returns. In this case, the Gaussian ellipsoids is based on a mean $(0.00,0.00)$ and a one-step-ahead covariance matrix is given by $\operatorname{vech}\left(\widehat{H}_{T+1 \mid T}\right)=(0.05,0.04,0.13)$, while the bootstrap ellipsoid is centered at $(0.00,0.01)$ and the bootstrap conditional covariance matrix is given by $\operatorname{vech}\left(\widehat{H}_{T+1 \mid T}^{*}\right)=(0.06,0.05,0.13)$. Clearly, these two moments are rather similar in both cases and the corresponding ellipsoids are also similar. Second, as expected, we note that the bootstrap corrected cubes are larger than those based on Gaussianity, a fact that might be attributed to the parameter uncertainty and the non-Gaussianity of the errors. In order to differentiate the effect of both features, we implemented a slight modification of the proposed bootstrap procedure, which consist in omitting the parameter uncertainty by fixing the parameter at their three-steps correlation target estimate throughout all bootstrap forecast replicates. As a result, we avoid the sampling variability due the parameter uncertainty while we are able to capture the 
asymmetries in the empirical distribution of the error. In this case, the difference between the bootstrap corrected cubes can be attributed to the parameter uncertainty. Figure 8 shows that both bootstrap cubes are rather similar. Consequently, the difference between the standard and bootstrap cubes is, to a large extent, due to the non-Gaussianity of the error with the parameter uncertainty playing a minor role. Once more, this is not surprising as the sample size in this empirical application is considerably large. As the financial market have reached a period of calm, extreme returns are less frequent during the out-of-sample period and, consequently, they tend to fall within the boundaries of all the regions plotted in Figure 8.

Finally, in order assess the adequacy of the out-of-sample $h$-steps-ahead bootstrap forecast intervals for correlations, we compute realized correlations; see Andersen et al. (2001), Andersen et al. (2003) and Barndorff-Nielsen and Shephard (2004). It is widely recognized that the estimation of realized covariances and correlations suffers from asynchronous trading and market microstructure noise, causing the covariance estimator to be biased and inconsistent; see, for instance, McAleer and Medeiros (2008) and Corsi and Audrino (2012). In our empirical example, the intra-day data is sampled using 1-minute intervals, avoiding the effect of non-synchronous data. The sample data spans from $2 / 8 / 2013$ to $13 / 9 / 2013$, which corresponds to 30 weekdays, and consist of transaction prices for the three exchange rates considered. In order to avoid the effect of non official trading we only consider prices between 9:00am and 4:00pm. After computing the intra-daily returns, the realized covariance matrix is obtained as follows

$$
H_{t}^{r}=\sum_{i=j}^{m} y_{(m) t+j / m} y_{(m) t+j / m}^{\prime}
$$

for $j=1, \ldots, m$ where $y_{(m) t+j / m}$ is the returns vector during the interval $\frac{1}{m}$; see Andersen et al. (2001) and Barndorff-Nielsen and Shephard (2004). Also, under some regularity assumption, the asymptotic distribution of the realized covariance and correlation matrices can be established; see Barndorff-Nielsen and Shephard (2004) for details. ${ }^{5}$

Figure 9 plots the $95 \%$ and $99 \% h$-steps-ahead bootstrap forecast intervals of correlations. The point forecast for the correlations of the cDCC model and realized correlations have also been plotted in Figure 9. We observe that the path depicted by the realized correlations is well-captured by their corresponding $95 \%$ and $99 \%$ bootstrap forecast intervals during the out-of-

\footnotetext{
${ }^{5}$ Dovonon et al. (2013) propose an i.i.d. bootstrap for realized correlations which is shown to outperform the asymptotic theory of Barndorff-Nielsen and Shephard (2004). Comparing the bootstrap intervals for daily correlations based on daily or high-frequency data is left for further research.
} 
sample period. Figure 9 also displays asymptotic $95 \%$ intervals of realized correlations computed using the results in Barndorff-Nielsen and Shephard (2004). We can observe that these intervals are mostly contained within the bootstrap intervals, suggesting that the latter depict reasonably well the uncertainty surrounding the conditional correlation estimates.

\section{Conclusion}

DCC models deliver within-sample and out-of-sample point forecasts of conditional correlations. However, uncertainty measures related to these forecasts are not available. In this paper, we propose to approximate the uncertainty of conditional correlations in the cDCC model by implementing the bootstrap procedure proposed by Fresoli et al. (2014) for multivariate models. We analyze its finite sample properties and show that it is adequate in situations similar to those encountered when fitting $\mathrm{cDCC}$ models to real systems of financial returns.

The bootstrap procedure is implemented to obtain predictive densities of returns, volatilities and correlations of a three-dimensional system of daily exchange rates returns. The bootstrap procedure seems to work adequately when forecasting out-of-sample in the sense that the bootstrap forecast intervals capture most of the realized correlations during the out-of-sample period.

Note also that the bootstrap procedure proposed in this paper can be easily adapted to deal with alternative MV-GARCH models; see Bauwens et al. (2006) and Silvennoinen and Teräsvirta (2009) for alternatives. In the context of VAR models, Fresoli et al. (2014) show that the advantages of bootstrapping are larger when the roots are close to the non-stationary regions, therefore it is of interest to analize the uncertainty associated with forecasting correlations in the context of non-stationary models as those suggested by Amado and Teräsvirta (in press).

Another important issue left for further research is the feasibility of the considered bootstrap procedure in large systems of returns. In this case, Hafner and Reznikova (2012) show that, in high dimensions, there is a problem of biased parameter estimates when implementing the three-steps target estimator considered in this paper due to the ill-conditioning of the sample correlation matrix, which is used for correlation targeting. They suggest using a shrinkage technique to solve this problem and compare it with alternative estimators. Alternatively, one can also use a bootstrap-after-bootstrap approach, though the computational burden is largely deepened. 


\section{References}

Aielli, G. P. (2013), "Dynamic conditional correlation: on properties and estimation," Journal of Business $\mathcal{E}$ Economic Statistics, 31, 282-299.

Aielli, G. P. and Caporin, M. (in press), "Variance clustering improved dynamic conditional correlation MGARCH estimators," Computational Statistics 8$\}$ Data Analysis.

Amado, C. and Teräsvirta, T. (in press), "Conditional correlation models of autoregressive conditional heteroskedasticity with nonstationary GARCH equations," Journal of Business 85 Economic Statistics.

Andersen, T. G., Bollerslev, T., Diebold, F. X., and Ebens, H. (2001), "The distribution of realized stock return volatility," Journal of Financial Economics, 61, 43-76.

Andersen, T. G., Bollerslev, T., Diebold, F. X., and Labys, P. (2003), "Modeling and forecasting realized volatility," Econometrica, 71, 579-625.

Arroyo, J. and Maté, C. (2009), "Forecasting histogram time series with k-nearest neighbours methods," International Journal of Forecasting, 25, 192-207.

Audrino, F. (in press), "Forecasting correlations during the late-2000s financial crisis: shortrun component, long-run component, and structural breaks," Computational Statistics \& Data Analysis.

Barndorff-Nielsen, O. E. and Shephard, N. (2004), "Econometric analysis of realized covariation: high frequency based covariance, regression, and correlation in financial economics," Econometrica, 72, 885-925.

Bauwens, L., Hafner, C. M., and Pierret, D. (2013), "Multivariate volatility modeling of electricity futures," Journal of Applied Econometrics, 28, 743-761.

Bauwens, L. and Laurent, S. (2005), "A new class of multivariate skew densities, with application to generalized autoregressive conditional heteroscedasticity models," Journal of Business $\&$ Economic Statistics, 23, 346-354.

Bauwens, L., Laurent, S., and Rombouts, J. V. (2006), "Multivariate GARCH models: a survey," Journal of Applied Econometrics, 21, 79-109.

Bollerslev, T. (1986), "Generalized Autoregressive Conditional Heteroskedasticity," Journal of Econometrics, 31, 307-327. 
Caporin, M. and McAleer, M. (2010), "Ranking multivariate GARCH models by problem dimension," Econometric Institute Research working paper series EI 2010-34.

— (2013), "Ten Things You Should Know About the Dynamic Conditional Correlation Representation," Econometrics, 1, 115-126.

Clark, T. E. and Ravazzolo, F. (in press), "Macroeconomic forecasting performance under alternative specifications of time-varying volatility," Journal of Applied Econometrics.

Corsi, F. and Audrino, F. (2012), "Realized covariance tick-by-tick in presence of rounded time stamps and general microstructure effects," Journal of Financial Econometrics, 10, 591-616.

Diebold, F. X. (1988), Empirical Modeling of Exchange Rate Dynamics, Springer New York.

Dovonon, P., Goncalves, S., and Meddahi, N. (2013), "Bootstrapping realized multivariate volatility measures," Journal of Econometrics, 172, 49-65.

Drost, F. C. and Nijman, T. E. (1993), "Temporal aggregation of GARCH processes," Econometrica, 61, 909-927.

Engle, R. F. (1982), "Autoregressive conditional heteroscedasticity with estimates of the variance of United Kingdom inflation," Econometrica, 50, 987-1007.

- (2002), "Dynamic Conditional Correlation," Journal of Business \& Economic Statistics, 20, $339-350$.

- (2009), Anticipating Correlations: A New Paradigm for Risk Management, Princeton University Press.

Engle, R. F. and Kelly, B. (2012), "Dynamic equicorrelation," Journal of Business \&3 Economic Statistics, 30, 212-228.

Engle, R. F. and Sheppard, K. (2001), "Theoretical and empirical properties of dynamic conditional correlation multivariate GARCH," Tech. rep., National Bureau of Economic Research.

Fiorentini, G., Sentana, E., and Calzolari, G. (2004), "On the validity of the Jarque-Bera Normality test in conditionally heteroskedastic dynamic regression models," Economics Letters, $83,307-312$.

Fresoli, D., Ruiz, E., and Pascual, L. (2014), "Bootstrap multi-step forecast of non-Gaussian VAR models," Manuscript. 
Gaglianone, W. P., Lima, L. R., Linton, O., and Smith, D. R. (2011), "Evaluating value-at-risk models via quantile regression," Journal of Business \& Economic Statistics, 29, 150-160.

Grigoletto, M. and Lisi, F. (2011), "Practical implications of higher moments in risk management," Statistical Methods \& Applications, 20, 487-506.

Hafner, C. M. and Franses, P. H. (2009), "A Generalized Dynamic Conditional Correlation model: simulation and application to many assets," Econometric Reviews, 28, 612-631.

Hafner, C. M. and Reznikova, O. (2012), "On the estimation of dynamic conditional correlation models," Computational Statistics $\&$ Data Analysis, 56, 3533-3545.

Hartz, C., Mittnik, S., and Paolella, M. (2006), "Accurate value-at-risk forecasting based on the normal-GARCH model," Computational Statistics \&3 Data Analysis, 51, 2295-2312.

Hong, Y., Tu, J., and Zhou, G. (2007), "Asymmetries in stock returns: statistical tests and economic evaluation," Review of Financial Studies, 20, 1547-1581.

Huang, T. H. and Wang, Y. H. (2012), "The volatility and density prediction performance of alternative GARCH models," Journal of Forecasting, 31, 157-171.

Jeon, J. and Taylor, J. W. (2012), "Using conditional kernel density estimation for wind power density forecasting," Journal of the American Statistical Association, 107, 66-79.

Laurent, S., Rombouts, J. V., and Violante, F. (2012), "On the forecasting accuracy of multivariate GARCH models," Journal of Applied Econometrics, 27, 934-955.

McAleer, M. and Medeiros, M. C. (2008), "Realized volatility: A review," Econometric Reviews, $27,10-45$.

Pascual, L., Romo, J., and Ruiz, E. (2006), "Bootstrap prediction for returns and volatilities in GARCH models," Computational Statistics \& Data Analysis, 50, 2293-2312.

Pesaran, B. and Pesaran, M. H. (2007), "Modelling volatilities and conditional correlations in futures markets with a multivariate t distribution," CESifo Working Paper No. 2056.

Rossi, E. and Spazzini, F. (2010), "Model and distribution uncertainty in multivariate GARCH estimation: a Monte Carlo analysis," Computational Statistics \& Data Analysis, 54, 2786-2800.

Rubner, Y., Tomasi, C., and Guibas, L. J. (1998), "A metric for distributions with applications to image databases," in Sixth International Conference on Computer Vision, IEEE, pp. 59-66. 
Silvennoinen, A. and Teräsvirta, T. (2009), "Multivariate GARCH models," in Handbook of Financial Time Series, Springer.

Teräsvirta, T. (2009), "An introduction to univariate GARCH models," in Handbook of Financial Time Series, Springer.

Wang, C.-P., Lin, S.-H., Huang, H.-H., and Wu, P.-C. (2012), "Using neural network for forecasting TXO price under different volatility models," Expert Systems with Applications, 39, $5025-5032$.

Wolf, M. and Wunderli, D. (2012), "Bootstrap joint prediction regions," University of Zurich Department of Economics Working Paper No. 64. 
Table 1: Summary statistics of daily returns for Euro (EUR), Japanese Yen (JPY) and Australian Dollar (AUD) against the US Dollar observed for the in-sample period from 3/1/2003 to 1/8/2013 (upper part), the out-of-sample period 2/8/2013 to 13/9/2013 (middel part) and the overall period from 3/1/2003 to $1 / 8 / 2013$. Mean, standard deviation (Std), skewnees (Skew) and excess kurtosis (Ek), Normality test (Fiorentini et al. (2004)), Ljung-Box for returns $(Q(10))$, squared returns $\left(Q_{2}(10)\right)$ and cross-correlations between returns and future squared returns $\left(Q_{12}(10)\right)$. Asymptotic $p$-values in parenthesis.

\begin{tabular}{|c|c|c|c|c|c|c|c|c|}
\hline & Mean & Std & Skew & Ek & Normality & $Q(10)$ & $Q_{2}(10)$ & $Q_{12}(10)$ \\
\hline \multicolumn{9}{|l|}{ Within-sample } \\
\hline EUR & 0.00 & 0.28 & $\begin{array}{l}-0.10 \\
(0.02)\end{array}$ & $\begin{array}{l}2.45 \\
(0.00)\end{array}$ & $\begin{array}{c}669.67 \\
(0.00)\end{array}$ & $\begin{array}{l}0.41 \\
(0.99)\end{array}$ & $\begin{array}{c}356.96 \\
(0.00)\end{array}$ & $\begin{array}{l}0.50 \\
(0.99)\end{array}$ \\
\hline JPY & 0.00 & 0.29 & $\begin{array}{l}-0.29 \\
(0.00)\end{array}$ & $\begin{array}{l}4.55 \\
(0.00)\end{array}$ & $\begin{array}{c}2324.29 \\
(0.00)\end{array}$ & $\begin{array}{l}0.53 \\
(0.99)\end{array}$ & $\begin{array}{c}164.39 \\
(0.00)\end{array}$ & $\begin{array}{l}3.25 \\
(0.97)\end{array}$ \\
\hline AUD & -0.01 & 0.40 & $\begin{array}{l}0.74 \\
(0.00) \\
\end{array}$ & $\begin{array}{l}12.22 \\
(0.00) \\
\end{array}$ & $\begin{array}{c}16761.15 \\
(0.00)\end{array}$ & $\begin{array}{l}2.88 \\
(0.98) \\
\end{array}$ & $\begin{array}{c}1665.31 \\
(0.00)\end{array}$ & $\begin{array}{l}80.95 \\
(0.00) \\
\end{array}$ \\
\hline \multicolumn{9}{|l|}{ Out-of-sample } \\
\hline EUR & -0.01 & 0.16 & $\begin{array}{l}0.24 \\
(0.30)\end{array}$ & $\begin{array}{l}-0.78 \\
(0.19)\end{array}$ & $\begin{array}{l}0.85 \\
(0.65)\end{array}$ & $\begin{array}{l}0.04 \\
(0.99)\end{array}$ & $\begin{array}{l}8.29 \\
(0.60)\end{array}$ & $\begin{array}{l}0.00 \\
(0.99)\end{array}$ \\
\hline JPY & 0.00 & 0.31 & $\begin{array}{l}0.10 \\
(0.41)\end{array}$ & $\begin{array}{c}-0.28 \\
(0.38)\end{array}$ & $\begin{array}{l}0.12 \\
(0.94)\end{array}$ & $\begin{array}{l}0.32 \\
(0.99)\end{array}$ & $\begin{array}{l}14.66 \\
(0.15)\end{array}$ & $\begin{array}{c}0.99 \\
(0.02)\end{array}$ \\
\hline AUD & -0.05 & 0.30 & $\begin{array}{l}-0.48 \\
(0.14) \\
\end{array}$ & $\begin{array}{l}-0.42 \\
(0.32) \\
\end{array}$ & $\begin{array}{l}1.14 \\
(0.56) \\
\end{array}$ & $\begin{array}{l}0.55 \\
(0.99) \\
\end{array}$ & $\begin{array}{l}7.85 \\
(0.64) \\
\end{array}$ & $\begin{array}{c}0.02 \\
(0.99) \\
\end{array}$ \\
\hline \multicolumn{9}{|l|}{ Overall period } \\
\hline EUR & 0.00 & 0.28 & $\begin{array}{l}-0.10 \\
(0.02)\end{array}$ & $\begin{array}{l}2.47 \\
(0.00)\end{array}$ & $\begin{array}{c}689.65 \\
(0.00)\end{array}$ & $\begin{array}{l}0.40 \\
(0.99)\end{array}$ & $\begin{array}{c}366.79 \\
(0.00)\end{array}$ & $\begin{array}{l}0.50 \\
(0.99)\end{array}$ \\
\hline JPY & 0.00 & 0.29 & $\begin{array}{l}-0.28 \\
(0.00)\end{array}$ & $\begin{array}{l}4.48 \\
(0.00)\end{array}$ & $\begin{array}{c}2285.50 \\
(0.00)\end{array}$ & $\begin{array}{l}0.54 \\
(0.99)\end{array}$ & $\begin{array}{c}164.05 \\
(0.00)\end{array}$ & $\begin{array}{l}3.17 \\
(0.98)\end{array}$ \\
\hline AUD & -0.01 & 0.40 & $\begin{array}{l}0.74 \\
(0.00)\end{array}$ & $\begin{array}{l}12.21 \\
(0.00)\end{array}$ & $\begin{array}{c}16945.48 \\
(0.00)\end{array}$ & $\begin{array}{l}2.82 \\
(0.99)\end{array}$ & $\begin{array}{c}1683.26 \\
(0.00)\end{array}$ & $\begin{array}{l}80.21 \\
(0.00)\end{array}$ \\
\hline
\end{tabular}


Table 2: Estimates of the parameters of the $\operatorname{cDCC}(1,1)$ model and diagnosis for daily returns of Euro (EUR), Japanese Yen (JPY) and Australian Dollar (AUD) exchange rates observed from $3 / 1 / 2003$ to $1 / 8 / 2013$. Ljung-Box statistic for the standardized squared residuals $\left(Q_{2}(10)\right)$, and multivariate Ljung-Box statistics for standardized residuals $\left(Q_{M}(10)\right)$. Asymptotic $p$-values in parenthesis.

\begin{tabular}{c|ccc|c}
\hline Conditional variances & $\omega_{i}$ & $\alpha_{i}$ & $\beta_{i}$ & $Q_{2}(10)$ \\
\hline EUR & $2.29 e^{-4}$ & 0.03 & 0.97 & 14.03 \\
& $(0.00)$ & $(0.00)$ & $(0.00)$ & $(0.170)$ \\
JPY & $8.37 e^{-4}$ & 0.03 & 0.96 & 10.64 \\
AUD & $(0.00)$ & $(0.00)$ & $(0.00)$ & $(0.39)$ \\
& $1.06 e^{-3}$ & 0.06 & 0.93 & 5.95 \\
& $(0.00)$ & $(0.00)$ & $(0.00)$ & $(0.82)$ \\
\hline Conditional correlations & $q_{i, j}$ & $\alpha$ & $\beta$ & $Q_{M}(10)$ \\
\hline EUR-JPY & 0.26 & - & - & - \\
EUR-AUD & $(0.00)$ & 0.04 & 0.95 & 93.30 \\
JPY-AUD & 0.63 & 0.04 & $(0.16)$ \\
& $(0.00)$ & $(0.00)$ & $(0.00)$ & - \\
& 0.11 & - & - & - \\
\hline
\end{tabular}




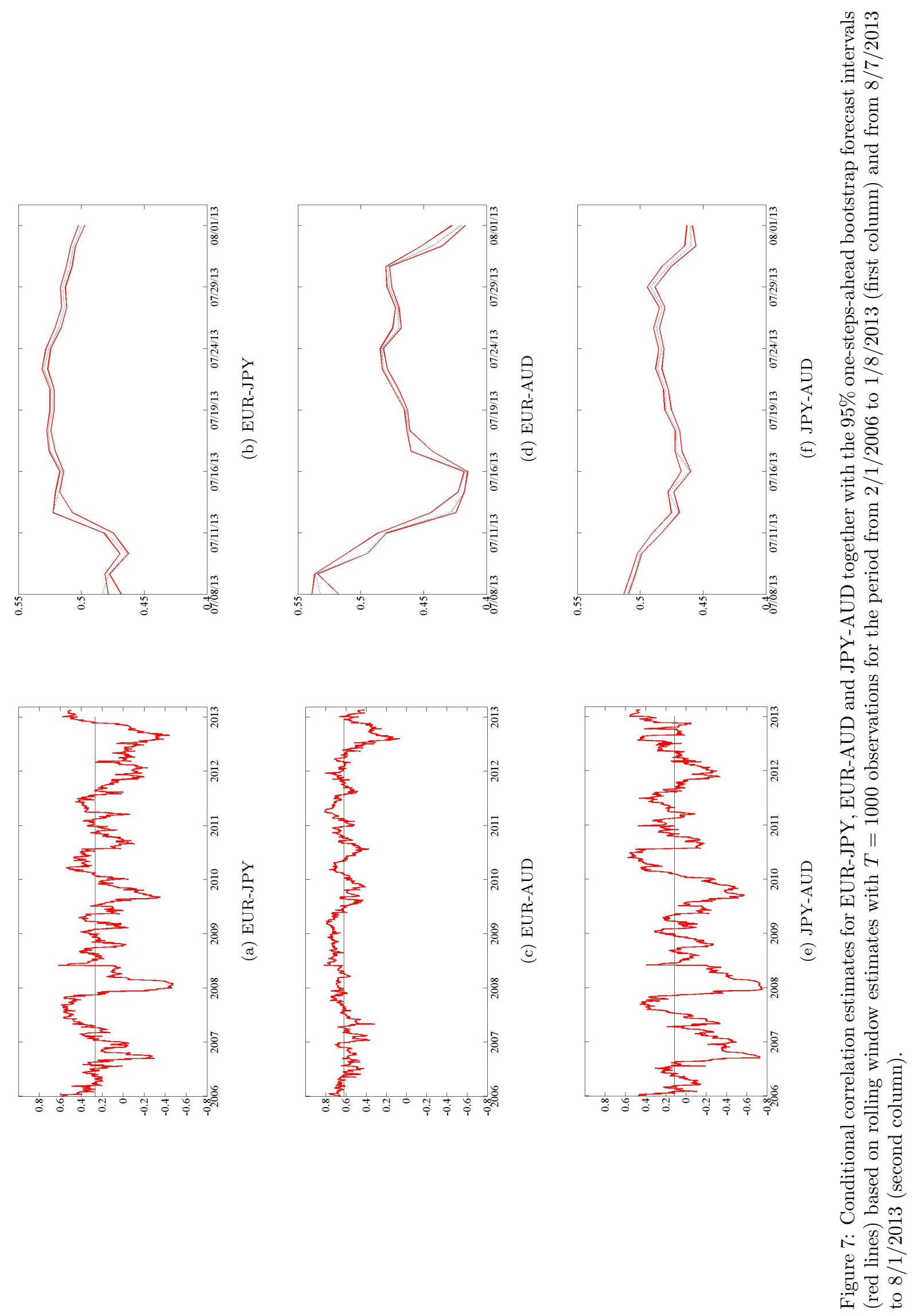



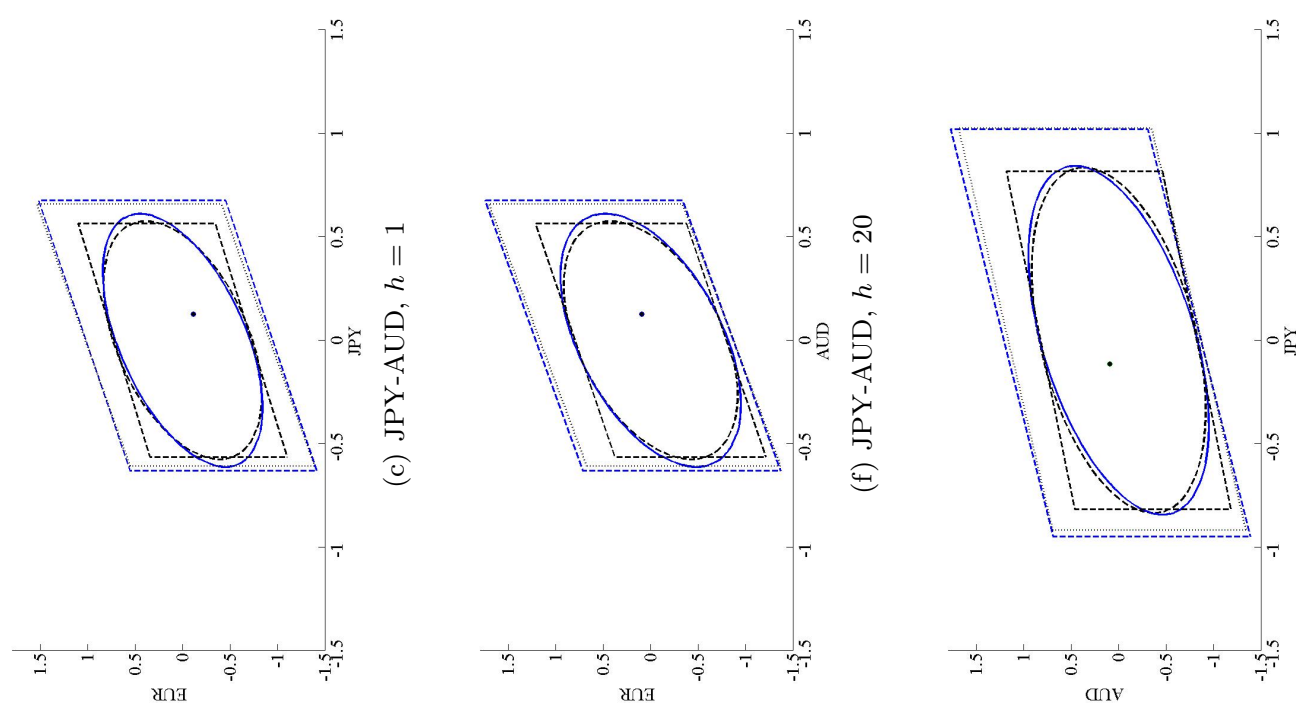

흉

:

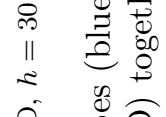

宽合

究 坖

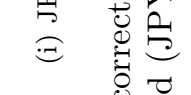

ชี

范

鱼实
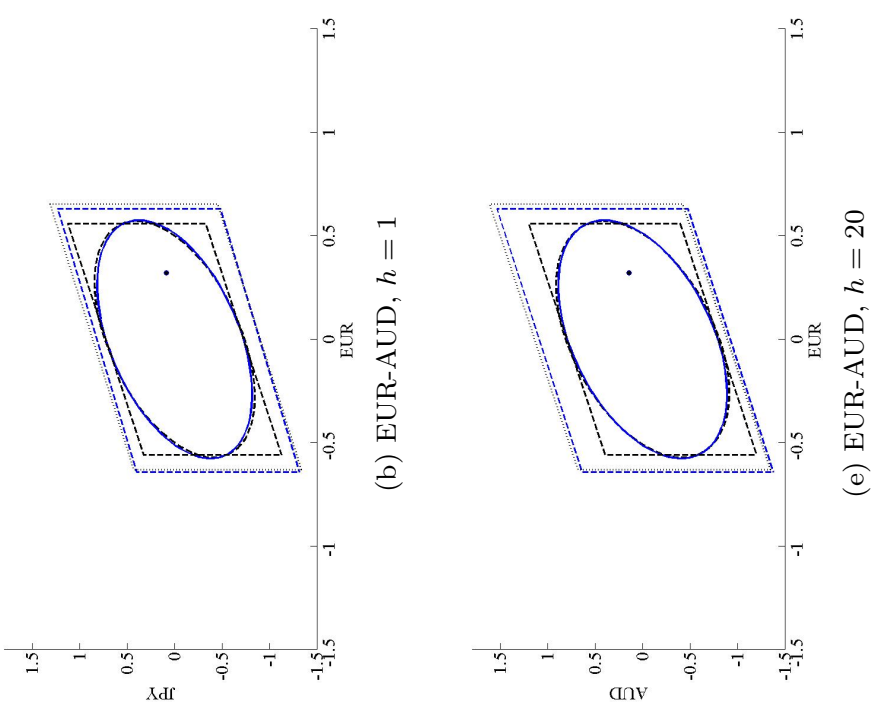

产

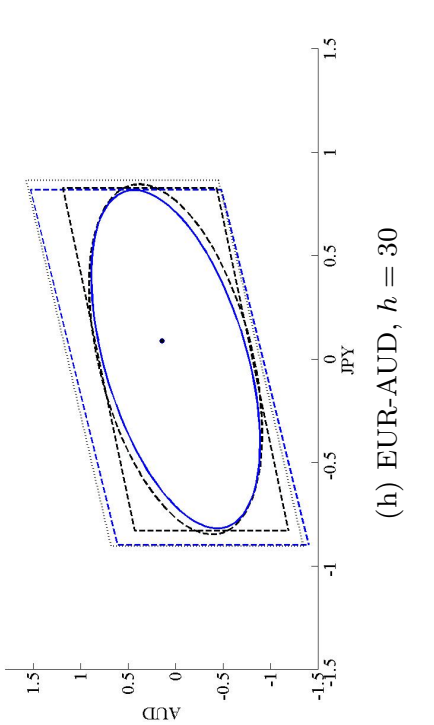

证

$\therefore$

国星

$\because$ ฮै

일

묭

:

过

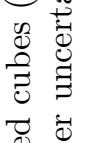

总

苍 :

节
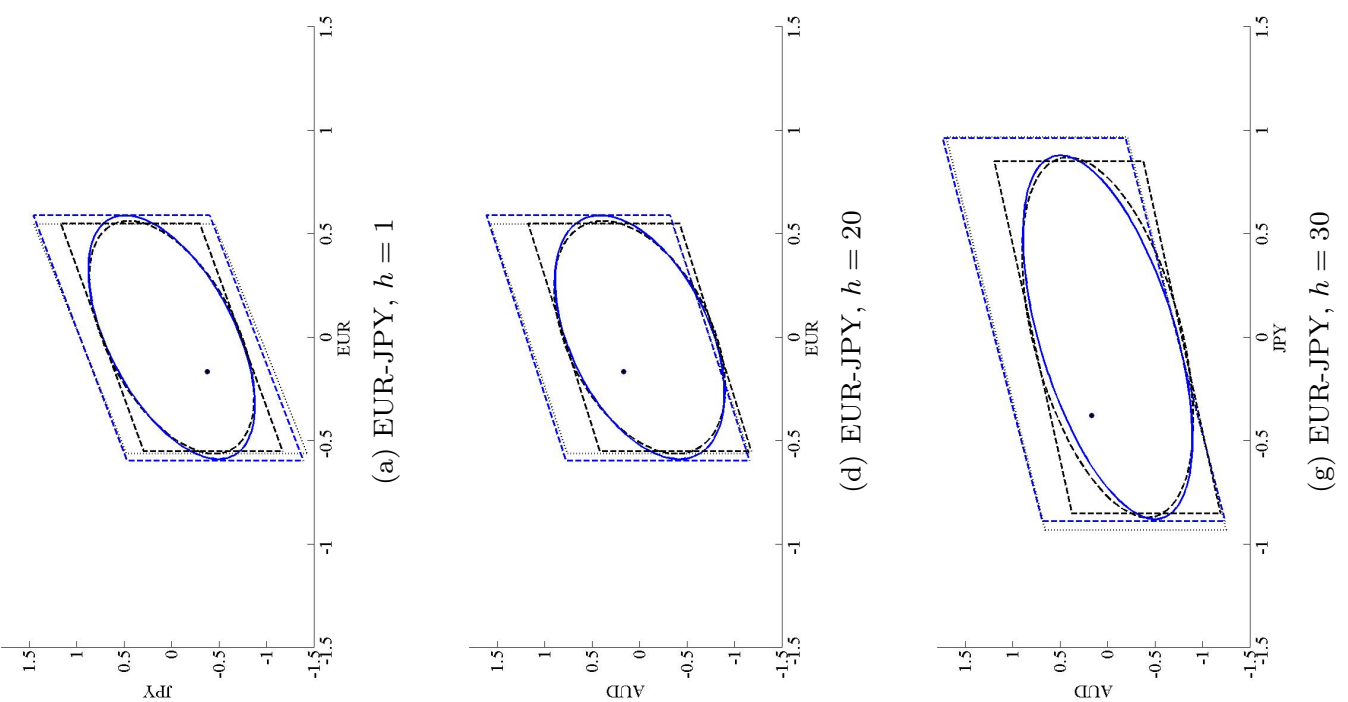

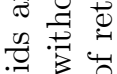

员.

항

. ఫี

क

ซึ :

ซ

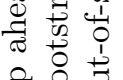

هิ응

点

일

요용

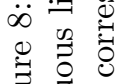

诖兽兽 


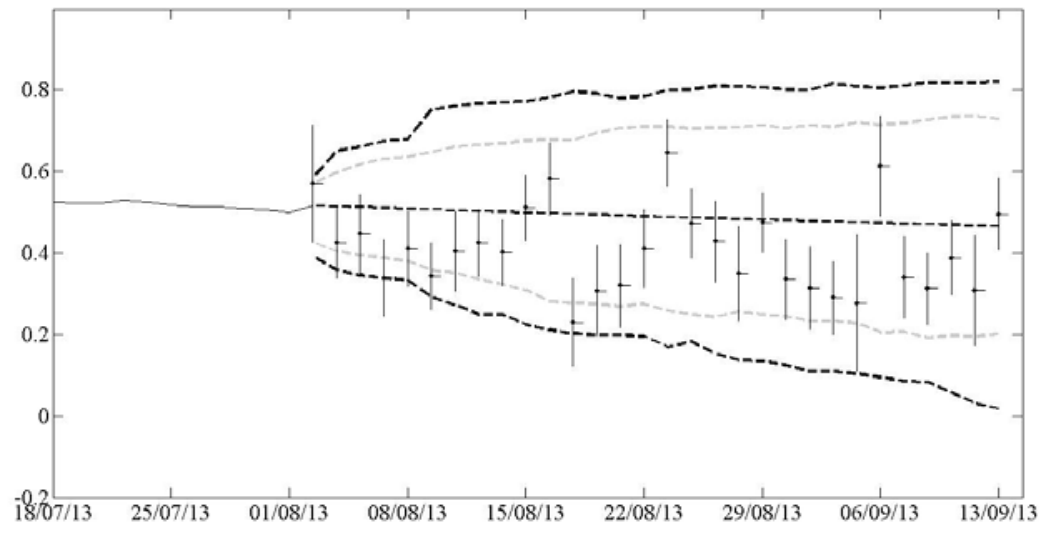

(a) EUR-JPY

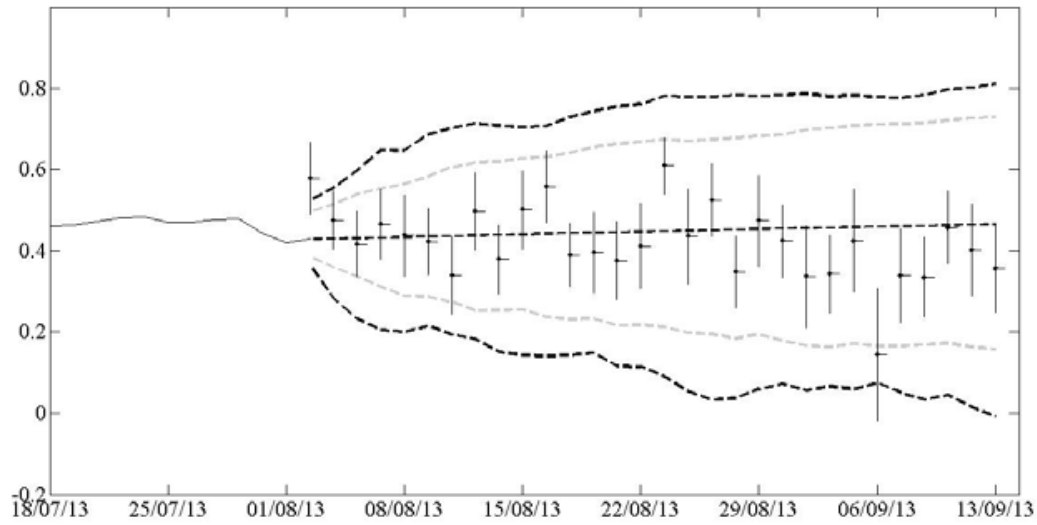

(b) EUR-AUD

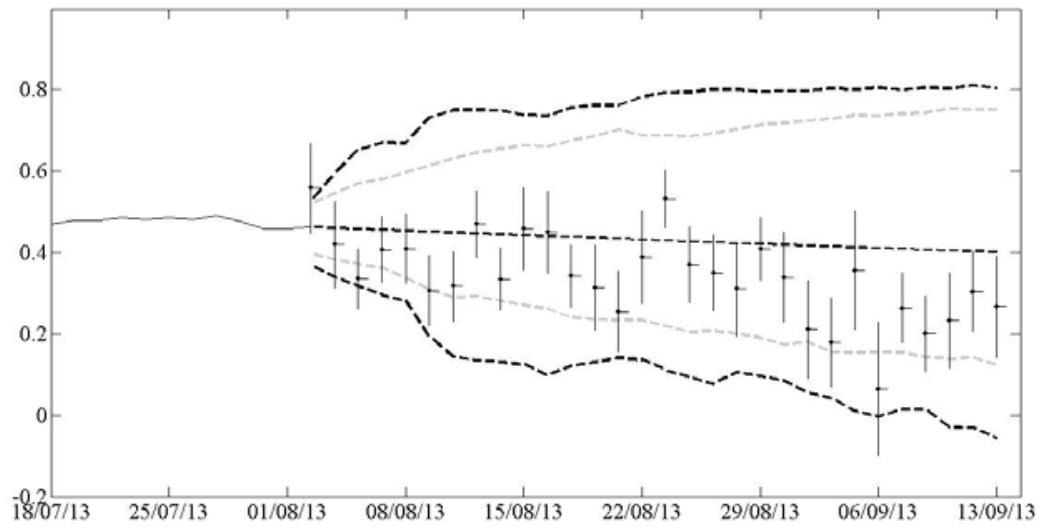

(c) JPY-AUD

Figure 9: Intra-daily realized correlations for EUR-JPY, EUR-AUD and JPY-AUD together with $95 \% h$-steps-ahead (grey discontinuos line) and $99 \%$ bootstrap $h$-steps-ahead (black discontinuos line) forecast intervals for the out-of-sample period from $2 / 8 / 2013$ to $13 / 9 / 2013$. The vertical lines represent $95 \%$ asymptotic intervals for realized correlations. 